\title{
Optical Sky Brightness and Transparency during the Winter Season at Dome A Antarctica from the Gattini-All-Sky Camera
}

\author{
Yi Yang ${ }^{1,2}$, Anna M. Moore ${ }^{3}$, Kevin Krisciunas ${ }^{1}$, Lifan Wang ${ }^{1,4,5}$, Michael C. B. Ashley ${ }^{6}$, Jianning Fu ${ }^{2}$, Peter J. Brown ${ }^{1}$, \\ Xiangqun Cui ${ }^{4,7}$, Long-Long Feng ${ }^{4,5}$, Xuefei Gong ${ }^{4,7}$, Zhongwen Hu ${ }^{4,7}$, Jon S. Lawrence ${ }^{8}$, Daniel Luong-Van ${ }^{6}$, \\ Reed L. Riddle ${ }^{9}$, Zhaohui Shang ${ }^{4,10}$, Geoff Sims ${ }^{6}$, John W. V. Storey ${ }^{6}$, Nicholas B. Suntzeff ${ }^{1}$, \\ Nick Tothill $^{11}$, Tony Travouillon ${ }^{9}$, Huigen Yang ${ }^{4,12}$, Ji Yang ${ }^{4,5}, \mathrm{Xu} \mathrm{Zhou}^{4,13}$, and Zhenxi Zhu ${ }^{4,5}$ \\ ${ }^{1}$ George P. and Cynthia Woods Mitchell Institute for Fundamental Physics \& Astronomy, Texas A. \& M. University, \\ Department of Physics \& Astronomy, 4242 TAMU, College Station, TX 77843, USA; ngc4594@physics.tamu.edu \\ ${ }^{2}$ Department of Astronomy, Beijing Normal University, Beijing 100875, P. R. China \\ ${ }^{3}$ Research School of Astronomy and Astrophysics, The Australian National University, Canberra, ACT 2611, Australia \\ ${ }^{4}$ Chinese Center for Antarctic Astronomy, Purple Mountain Observatory, Chinese Academy of Sciences, Nanjing 210008, P. R. China \\ ${ }^{5}$ Purple Mountain Observatory, Chinese Academy of Sciences, Nanjing 210008, P. R. China \\ ${ }^{6}$ School of Physics, University of New South Wales, Sydney, NSW 2052, Australia \\ ${ }^{7}$ Nanjing Institute of Astronomical Optics \& Technology, National Astronomical Observatories, \\ Chinese Academy of Sciences, Nanjing 210042, P. R. China \\ ${ }^{8}$ Australian Astronomical Observatory, Sydney, NSW 2113, Australia \\ ${ }^{9}$ California Institute of Technology, Pasadena, CA 91125, USA
${ }^{10}$ Tianjin Astrophysics Center, Tianjin Normal University, Tianjin 300387, P. R. China \\ ${ }^{11}$ School of Computing, Engineering \& Mathematics, University of Western Sydney, NSW 2751, Australia \\ ${ }^{12}$ Polar Research Institute of China, Shanghai 200136, P. R. China \\ ${ }^{13}$ National Astronomical Observatories, Chinese Academy of Science, Beijing 100012, P. R. China \\ Received 2016 October 30; revised 2017 May 13; accepted 2017 May 15; published 2017 June 13
}

\begin{abstract}
The summit of the Antarctic plateau, Dome A, is proving to be an excellent site for optical, near-infrared, and terahertz astronomical observations. Gattini is a wide-field camera installed on the PLATO instrument module as part of the Chinese-led traverse to Dome A in 2009 January. We present here the measurements of sky brightness with the Gattini ultra-large field of view $\left(90^{\circ} \times 90^{\circ}\right)$ in the photometric $B$-, $V$-, and $R$-bands; cloud cover statistics measured during the 2009 winter season; and an estimate of the sky transparency. A cumulative probability distribution indicates that the darkest $10 \%$ of the nights at Dome A have sky brightness of $S_{B}=22.98$, $S_{V}=21.86$, and $S_{R}=21.68 \mathrm{mag} \operatorname{arcsec}^{-2}$. These values were obtained during the year 2009 with minimum aurora, and they are comparable to the faintest sky brightness at Maunakea and the best sites of northern Chile. Since every filter includes strong auroral lines that effectively contaminate the sky brightness measurements, for instruments working around the auroral lines, either with custom filters or with high spectral resolution instruments, these values could be easily obtained on a more routine basis. In addition, we present example light curves for bright targets to emphasize the unprecedented observational window function available from this ground-based site. These light curves will be published in a future paper.
\end{abstract}

Key words: methods: data analysis - methods: statistical - site testing - techniques: photometric - telescopes

\section{Introduction}

Over the past centuries, the growing demand for large astronomical facilities keeps pushing forward the progress of astronomical site selections. Some of the most major considerations for ground-based optical and IR astronomy include the seeing, atmospheric transparency and cloud coverage, number of clear nights, wind speed, precipitable water vapor, and thermal backgrounds. Various site surveys in recent years have revealed the advantages of the Antarctic plateau sites. Low and stable count rates of sky backgrounds in optical bandpasses have been measured at Dome C (Kenyon \& Storey 2006), the south pole (Ashley et al. 1996; Nguyen et al. 1996), and Dome A (Zou et al. 2010; Sims et al. 2012a). High atmospheric transmission has been inferred at Dome A (Lawrence 2004; Yang et al. 2009) and various Antarctic sites (Lawrence 2004). Good average optical seeing above the boundary layer can be obtained at the south pole, i.e., 0" 37 at $\lambda=2000 \AA$ at $17-27 \mathrm{~m}$ above the ground (Marks et al. 1996), and $0 . \prime 23$ at $\lambda=2.4 \mu \mathrm{m}$ (Marks 2002). At Dome C, Lawrence et al. (2004) found seeing of 0"'27, while Agabi et al. (2006) determined 0 "! $36 \pm 0$ !" 19 , Aristidi et al. (2009) found 0!"36, and Giordano et al. (2012) found $<0 ! 3$ at $\sim 30 \mathrm{~m}$ above the ground. See also Vernin et al. (2009) and Aristidi et al. (2015). Many astronomical observations could benefit from the consecutive periods of dark time at polar latitudes. Comparisons among the different astronomical sites in the Antarctic plateau are being made regarding cloud coverage, aurorae, thickness of the boundary layer, seeing, humidity, and temperature (Saunders et al. 2009).

The high-altitude Antarctic sites of Dome A (latitude $80^{\circ} 22^{\prime} \mathrm{S}$, longitude $77^{\circ} 21^{\prime} \mathrm{E}$, elevation $4093 \mathrm{~m}$ ) offer intriguing locations for future large-scale astronomical observatories. Site testing work since 2008 has confirmed Dome A to be an excellent astronomical site. The extremely thin turbulent boundary layer measured to be $13.9 \mathrm{~m}$ near the ground at Dome A enables a free-of-atmosphere observing condition for a telescope on a small tower (Bonner et al. 2010). Some other advantages include the low sky brightness measured in the SDSS $i$-band (Zou et al. 2010), the outstanding low cloud coverage compared to other astronomical sites (Zou et al. 2010), and the extremely low atmospheric water vapor content (Sims et al. 2012b). Additionally, the airglow and 
aurorae at Dome A in the optical and near-IR range during the 2009 winter season have been characterized by Sims et al. (2012b), and only $2 \%$ of the time during 2008 winter season (solar minimum) have shown strong auroral events in the $i$-band (Zou et al. 2010). Dome A also has exceptional transmission and multi-day persistent superlative observing conditions in the terahertz regime (Yang et al. 2010).

Time-series photometry has long been an essential tool to study the stellar properties as well as other astrophysical phenomena involving time-variant celestial objects. Long-term monitoring of stars to a very high degree of precision probes a wide range of frequencies. Over the last decades, there has been rapid progress in consecutive, high-quality, and high-cadence transiting surveys. Space-based missions including the Swift Ultraviolet/Optical Telescope (Gehrels et al. 2004; Roming et al. 2005) is leading the high-energy regime; the CoRoT satellite (Baglin et al. 2006) and the Kepler satellite (Borucki et al. 2010) are searching for Earth-size planets and performing asteroseismology on field stars. A summary of ground-based untargeted transient and variable surveys can be found in Table 1 of Rau et al. (2009).

Consecutive monitoring for as long as months is not possible at single-site mid-latitude astronomical observatories. Although space-based transient surveys achieve better accuracy due to the extremely low atmospheric absorption, turbulence, and light pollution, ground-based sites offer advantages such as unlimited cadence, flexible pointing, and the feasibility of following brighter targets. Taking the advantages of the long "winter night" as well as other remarkable observation conditions at Dome A, highquality time-series observations carried out with small telescopes have already been obtained, opening a window for asteroseismology at the Antarctica plateau sites.

Previous works demonstrated that high photometric accuracy can be acquired by small aperture telescopes at Dome A. The first comprehensive studies on asteroseismology and stellar physics at Dome A Antarctica were conducted by the $14.5 \mathrm{~cm}$ diameter Chinese Small Telescope ARray (CSTAR; Yuan et al. 2008), which has monitored an area around the South Celestial Pole since 2008. The nature of the CSTAR wide field design (field of view $(\mathrm{FOV})$ of $4.5 \times 4.5)$ and the absence of a sidereal tracking system introduced significant systematic uncertainties into the stellar photometry. Various efforts have been made to reduce the systematic errors and to push the photometric precision below a few mmag, including the modeling of the inhomogeneous effects of clouds (Wang et al. 2012), the ghost images (Meng et al. 2013), and the systematic diurnal residuals (Wang et al. 2014b). Based on the detrended light curves obtained during the 2008 winter season, comprehensive studies on exoplanet candidates (Wang et al. 2014a), stellar variability (Wang et al. 2015), eclipsing binaries (Yang et al. 2015), and stellar flares (Liang et al. 2016) were carried out. Other independent studies include those on variable sources (Wang et al. 2011, 2013; Oelkers et al. 2015), and specific studies on the pulsation modes of RR Lyrae stars (Huang et al. 2015) and $\delta$ Scuti variables (Zong et al. 2015) based on the CSTAR observations in single or multiple years from 2008 through 2010. We also note that variable stars were observed during the 2012 winter season ( $\mathrm{Li}$ et al. 2015) with a single unit of the Antarctic Survey Telescopes (AST3; Yuan et al. 2014). In addition, time-series photometry studies have also been conducted at Dome C Antarctica, for instance, the careful time-series multicolor photometry to study stellar pulsation and evolution using the Photometer AntarctIca eXtinction (PAIX; Chadid et al. 2010, 2014, 2016).

These transient surveys at Antarctic sites with $\sim 10 \mathrm{~cm}$ class and larger telescopes have measured stars within a magnitude range of $\sim 8$ to $\sim 15$ with an FOV of $\lesssim 20$ square degrees. The multiband ultra-wide FOV imaging obtained by the GattiniDome A camera during the 2009 winter season is also a valuable data set considering its continuous monitoring of the flux variation of the third to seventh magnitude stars. Multiband photometric results for bright targets obtained during the 2009 winter season by the Gattini-Dome A camera will be discussed in a future paper.

In this paper, we analyze a multiwavelength data set collected at Dome A Antarctica during the 2009 winter season. We have measured and calibrated the sky brightness in three photometric bands. We estimate the transparency variations and perform aperture photometry for those targets in the $V$ magnitude range $\sim 3.5$ to $\sim 7.5$. We focus on targets in the sky south of decl. $-50^{\circ}$. The organization of this paper is as follows. In Section 2, we describe the instrument and observations. Section 3 discusses the data reduction pipeline. In Section 4, we give our results, and in Section 5 our conclusions.

\section{Importance of Sky Background Measurements}

When doing astronomical photometry, the ideal result is to determine the brightness of individual celestial objects. However, various sources including the scattered light from the Sun, the Moon, the aurora, and airglow will contaminate the flux from the astronomical sources. A summary of the contributions to the light of the night sky has been provided by Roach \& Gordon (1973) and Kenyon \& Storey (2006). Considering the case of aperture photometry, the total flux integrated over the circular aperture can be expressed as Flux $=$ Source $+\mathrm{Sky}+\mathrm{RN}^{2}+$ Dark. The terms on the right-hand side are the source counts enclosed by the aperture from a celestial object, the sky background, the readout noise $\left(\mathrm{RN}^{2}\right)$, and the electron counts from the dark current, respectively. Due to the discrete nature of the electric charge, a Poisson process models the counting process of the photons, and the error is given by the square root of the total counts. By adding the noise terms in quadrature, the signal-to-noise ratio $(\mathrm{S} / \mathrm{N})$ for an object measured in an aperture with radius $r$ can be written as $\mathrm{S} / \mathrm{N}=$ Source $/ \sqrt{\text { Source }+\mathrm{Sky}+\mathrm{RN}^{2}+\text { Dark }}$. In a sky-limited case, i.e., $\sqrt{\mathrm{Sky}}>3 \mathrm{RN}, \mathrm{S} / \mathrm{N} \approx$ Source $/ \sqrt{\mathrm{Sky}}$. The temporal variation of the sky background significantly influences the efficiency and detection capability of ground-based astronomical facilities, especially in the low- $\mathrm{S} / \mathrm{N}$ regime.

Given the critical role played by sky brightness in astronomical site selection, the measurement of the background light of the sky has been carried out for over a century using photographic plates, photomultiplier tubes, and modern digital detectors. A classic monograph on the subject is The Light of the Night Sky by Roach \& Gordon (1973). A comprehensive summary of the published sky background statistics is given by Benn \& Ellison (1998). Some useful background can also be found in papers by Walker (1988), Krisciunas (1997), Patat (2003), Krisciunas et al. (2007), and references therein.

The daily and monthly variations of the sky brightness at the high-altitude Antarctic Dome A site are expected to be different from those of mid-latitude sites. It can be shown that the angle of solar elevation, $\alpha$, can be approximated in terms of the solar decl. angle on a given date $\delta(d)$, the hour angle of 
observation at a given time during the day $h(t)$, and the observer's latitude $\phi$ :

$$
\alpha=\sin ^{-1}(\sin \delta(\boldsymbol{d}) \sin \phi+\cos \delta(\boldsymbol{d}) \cos [\boldsymbol{h}(\boldsymbol{t})] \cos \phi) .
$$

The Sun's decl. can be approximated by

$$
\delta=23.45 \times \sin \left[\frac{2 \pi}{365} \times(d+284)\right]
$$

where $d$ is the day of the year with January 1 as $d=1$; given $\phi=-80^{\circ} 22^{\prime}$ for the Dome A site, on the summer solstice $(d=172)$, the Sun obtains its lowest mean altitude over a sidereal day, i.e., $h(t)$ from 0 to $24 \mathrm{hr}, \alpha$ ranges from $-13^{\circ} .8$ to $-33^{\circ}$. 1 . Therefore, even continual darkness can be expected during the winter season at Antarctic sites; however, the latitude of Dome A never allows the Sun to stay more than $18^{\circ}$ below the horizon for an entire sidereal day. The twilight due to the scattered light from the Sun and the Moon without the additional scattering by clouds needs to be modeled to better understand the scattering effect of the atmosphere at Dome A. For other sites, Krisciunas \& Schaefer (1991) present a model of the $V$-band sky brightness when there is moonlight. Liu et al. (2003) also present a model of the brightness of moonlight as a function of lunar phase angle and elevation above the horizon. A much more advanced moonlight model, based on spectra taken at Cerro Paranal, Chile, is presented by Jones et al. (2013).

\section{Instrument and Observations}

\subsection{Project Goals}

The multiband sky brightness at Dome A, as well as the sky brightness in a large area of the night sky, is an unknown quantity. The Gattini project was created to unambiguously measure the optical sky brightness within an incredibly large $90^{\circ} \times 90^{\circ} \mathrm{FOV}$, as well as the cloud coverage and aurora of the winter-time sky above such a high-altitude Antarctic site. The Gattini-Dome A All-Sky Camera (GASC; Moore et al. 2008) was installed on the PLATO (PLATeau Observatory) instrument module, which is an automated self-powered astrophysical observatory deployed to Dome A (Yang et al. 2009) as part of the Chinese-led expedition to the highest point on the Antarctic plateau in 2008 January. This single automated wide-field camera contains a suite of Bessell photometric filters $(B, V, R)$ and a longpass red filter for the detection and monitoring of $\mathrm{OH}$ emission. We have in hand one complete winter-time data set (2009) from the camera that was returned in 2010 April. The extremely large FOV of the GASC allows us to monitor the night sky brightness in the $B, V$, and $R$ photometric bands and the cloud cover beginning in the 2009 winter season at Dome A over a wide range of zenith angles $\left(0^{\circ}\right.$ to $\left.\sim 30^{\circ}\right)$. Multiband sky intensities measured by GASC in combination with spectra obtained with the NIGEL instrument (Sims et al. 2010) will offer more comprehensive statistics on aurora and airglow. In addition, photometry of bright target stars in the GASC FOV with an unprecedented temporal window function is permitted by months of continual darkness during the Antarctic winter. An overview of the multiband GASC FOV is shown in Figure 1.

\subsection{Dome A Camera Design and Assembly}

The GASC was a novel low-cost pathfinder that ambitiously set out to measure the multiyear sky properties of one of the most remote and desolate sites on the planet. It was assembled at Caltech in 2008 and consists of a Nikon $10.5 \mathrm{~mm} \mathrm{f/2.8} \mathrm{GED}$ DX fisheye lens mated to an Apogee Alta U4000 $2 \mathrm{~K} \times 2 \mathrm{~K}$ interline camera, and a filter wheel with an assortment of photometric filters. The system is housed inside a heated enclosure and controlled by a rugged PC-based supervisor system. The project cost, including labor for fabrication and test but excluding logistical costs, was approximately $\$ 50,000$ US dollars (in 2008). The system was pointing near the SCP, without guiding or field rotation. The system gives a mean plate scale of approximately $150^{\prime \prime}$ pixel $^{-1}$, about $147^{\prime \prime} \mathrm{pixel}^{-1}$ near the center of the FOV and $\sim 155^{\prime \prime}$ pixel $^{-1}$ near the edges of the FOV. The entire field is about $85^{\circ} \times 85^{\circ}$. In between the lens and the camera is a five-position filter wheel containing Bessell $B, V$, and $R$ filters (Bessell 1990). The remaining two slots are for a longpass red filter $(\lambda>650 \mathrm{~nm})$ for the study of airglow $(\mathrm{OH})$ emission and an opaque mask for dark current tests.

The heat permits successful operation of the off-the-shelf camera as well as preventing and eradicating ice on the window surface. A conductive indium tin oxide coating was employed on the window surface as a method of de-icing. However, it was found that when operational, this was not sufficient to remove ice that was deposited in large amounts due to the surface wind. Some images showed very few sources over small uninterrupted periods of time, ranging from hours up to $\sim 3$ days. It is not possible to determine whether the lack of astronomical sources was caused by ice and frost formed on the cover window, or because of the atmosphere. The internal heating was sufficient to keep the window ice-free during the rest of the winter period.

The experiment was controlled by a low-power computer in an electronics rack inside the PLATO module. The continuous observation was operationally simple and repeated this sequence: $B$-band (100 and $30 \mathrm{~s}), V$-band (100 and $30 \mathrm{~s}), R$-band (100 and $30 \mathrm{~s}$ ), OH-band as a longpass red filter (100 and $30 \mathrm{~s}), 100 \mathrm{~s}$ dark, and bias frame. During periods of bright twilight at the beginning and end of the 2009 winter season, the camera adjusted its exposure time to adapt to the sky counts, or truncated integrations to prevent overexposure.

\subsection{The 2009 Data Set}

The full data set contains approximately 160,000 images obtained from 2009 April 18 to October 10. Unfortunately, the images obtained before 2009 May 19 were affected by snow over more than one-third of the FOV. Considering the unknown transmission and reflection caused by the anisotropic snow coverage, those images were discarded. Additionally, the sky became continuously bright due to the Sun after 2009 September 18, so we excluded from the data reduction all the images obtained after that date. Images obtained prior to that date, however, with the Sun's elevation angle greater than $-10^{\circ}$ and the median count rate above a certain threshold, have also been excluded. Images with a $100 \mathrm{~s}$ exposure time have been used to inspect and calibrate the sky brightness. Approximately 11,925 frames were obtained in each photometric band for each exposure time between 2009 May 19 and September 18 ( $\sim 123$ days), resulting in a total of $331.25 \mathrm{hr}$ of 


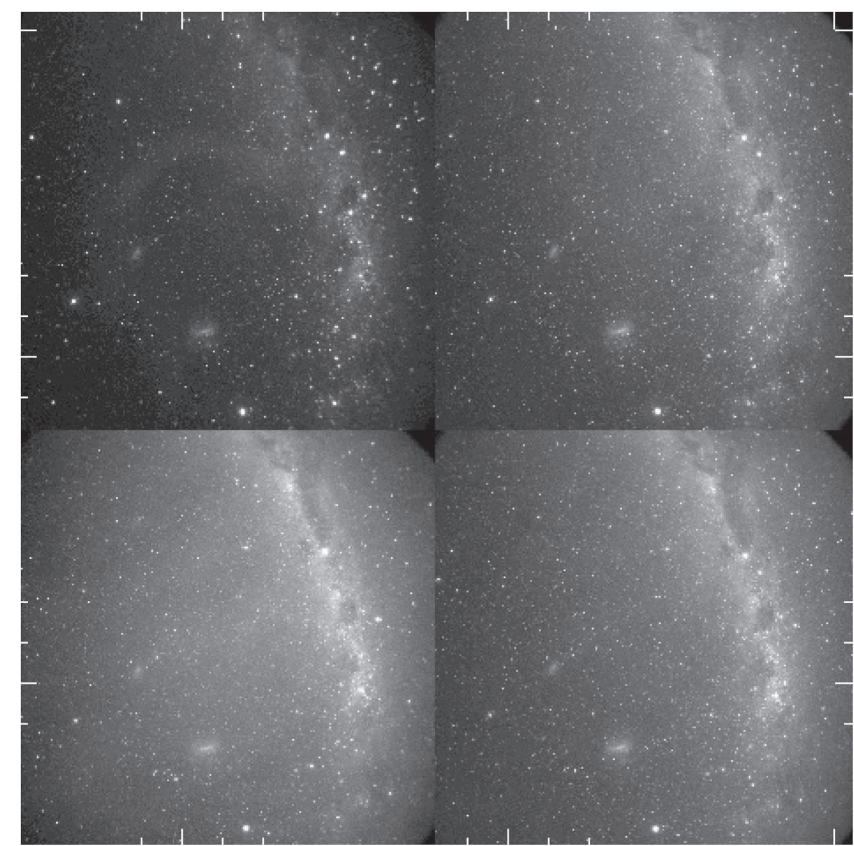

Figure 1. Multiband images obtained by GASC on 2009 June 21. The upperleft, upper-right, lower-right, and lower-left panels present the $B-, V-, R-$, and $\mathrm{OH}$-band images, respectively. The Milky Way runs from the top middle toward the lower right in each panel, and the LMC and SMC can be identified in the lower-left quadrant of each panel.

$100 \mathrm{~s}$ exposures in each filter. The typical cadence for the $B$-, $V$-, and $R$-band $100 \mathrm{~s}$ exposure sequences requires $\sim 737 \mathrm{~s}$.

\subsection{Instrumental Effects}

GASC was set out to measure the multiyear sky properties of one of the most remote and desolate sites on the planet. Given the nature of the wide field design, combined with an interline CCD and no sidereal tracking system, non-negligible effects needed to be modeled to process the data effectively. These effects are summarized as follows.

\subsubsection{Absence of a Sidereal Tracking System}

The camera was mounted in a heated enclosure, with fixed pointing in the direction of the SCP. The absence of a mechanical tracking system, together with an extremely large FOV, produces stellar images that exhibit a different Point Spread Function (PSF) at different positions throughout the FOV. Over the course of the $100 \mathrm{~s}$ exposure, the stars produced elongated circular tracks owing to the Earth's rotation. This effect is most obvious for stars farthest from the exact location of the SCP, which was close to the center of the GASC FOV. The observed largest elongations are $\sim 6$ pixels in the $X$ direction and $\sim 6$ pixels in the $Y$ direction for each $100 \mathrm{~s}$ exposure frame.

\subsubsection{Angle between the Optical Axis and the South Celestial Pole}

The optical axis of the camera was closely aligned with the SCP, while the horizontal axis of the cover window of the heated enclosure was fixed to be aligned with the zenith. Because stars obtain different zenith angles as a result of the rotation of the Earth, both the optical path length through the material of the cover window and the thickness of the Earth's atmosphere

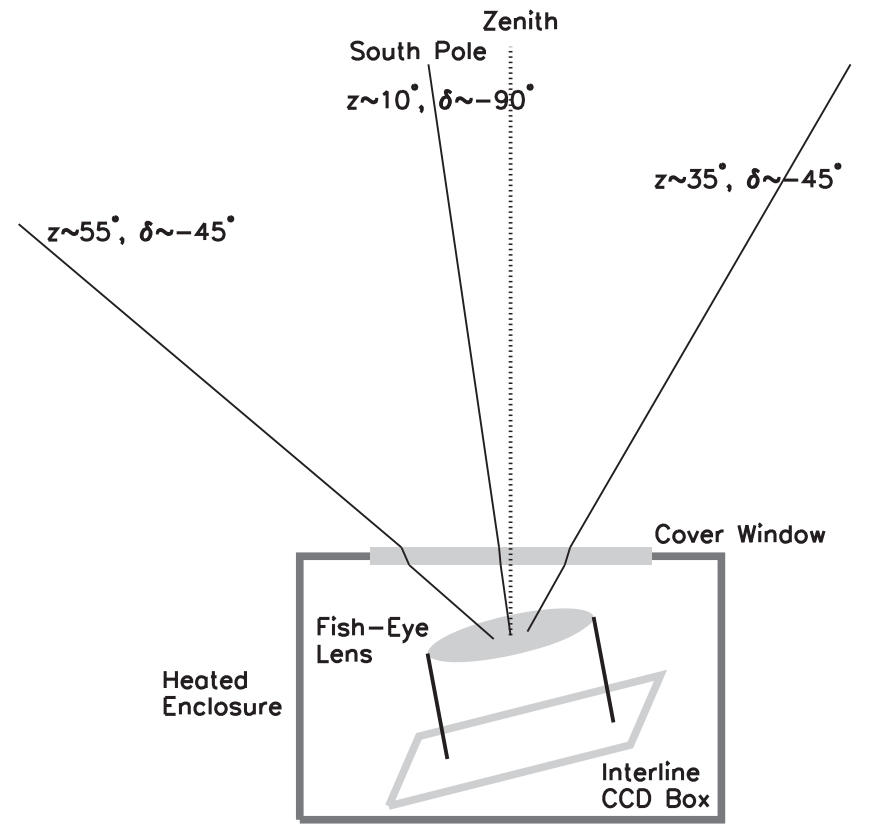

Figure 2. Schematic diagram showing the setup of GASC.

changes with stellar azimuths and elevation angles, introducing a periodic, asymmetrical variation of the stellar light curves, even for stars of constant brightness. Additionally, since the GASC camera was pointing near the SCP, while the cover window was pointing at the zenith, the $\sim 10^{\circ}$ offset between the SCP and zenith introduced transmission differences as the light passed through the cover window. A schematic of this setup is presented in Figure 2.

\subsubsection{Vignetting}

In large-field astronomical images, as well as in photography and optics, vignetting causes a reduction of flux at the periphery compared to the image center. In the optical design of GASC, vignetting was necessary to minimize the scattered light from the Moon when it is above the horizon. However, vignetting also significantly reduces both the flux from the stars and from the sky background, especially at the edges of the GASC FOV.

\subsubsection{Interline Transfer Sensor}

The detector situated behind the multiband filter wheel is a $2 \mathrm{~K} \times 2 \mathrm{~K}$ interline transfer $\mathrm{CCD}$. It has a parallel register that has been subdivided into two stripes to create opaque storage register fits between each pair of columns of pixels. These opaque masks occupy a large portion of the area of the CCD. Although micro-lenses that focus light from a large area down to the photodiode have been annealed to the CCD, when light beams are incident at large angles, the micro-lens array will fail to direct all the photons directly down to the photodiode. For this reason, the CCD is less sensitive to some incident directions of light. As the stars move around the SCP, this effect will cause periodic fluctuations in the resulting light curves. The amplitude of those variations is strongly correlated with a star's angular distance from the SCP. For the GASC optical system, this effect can reach $\sim 0.2$ mag. Further test and analysis will be presented in Section 4.4.3. 


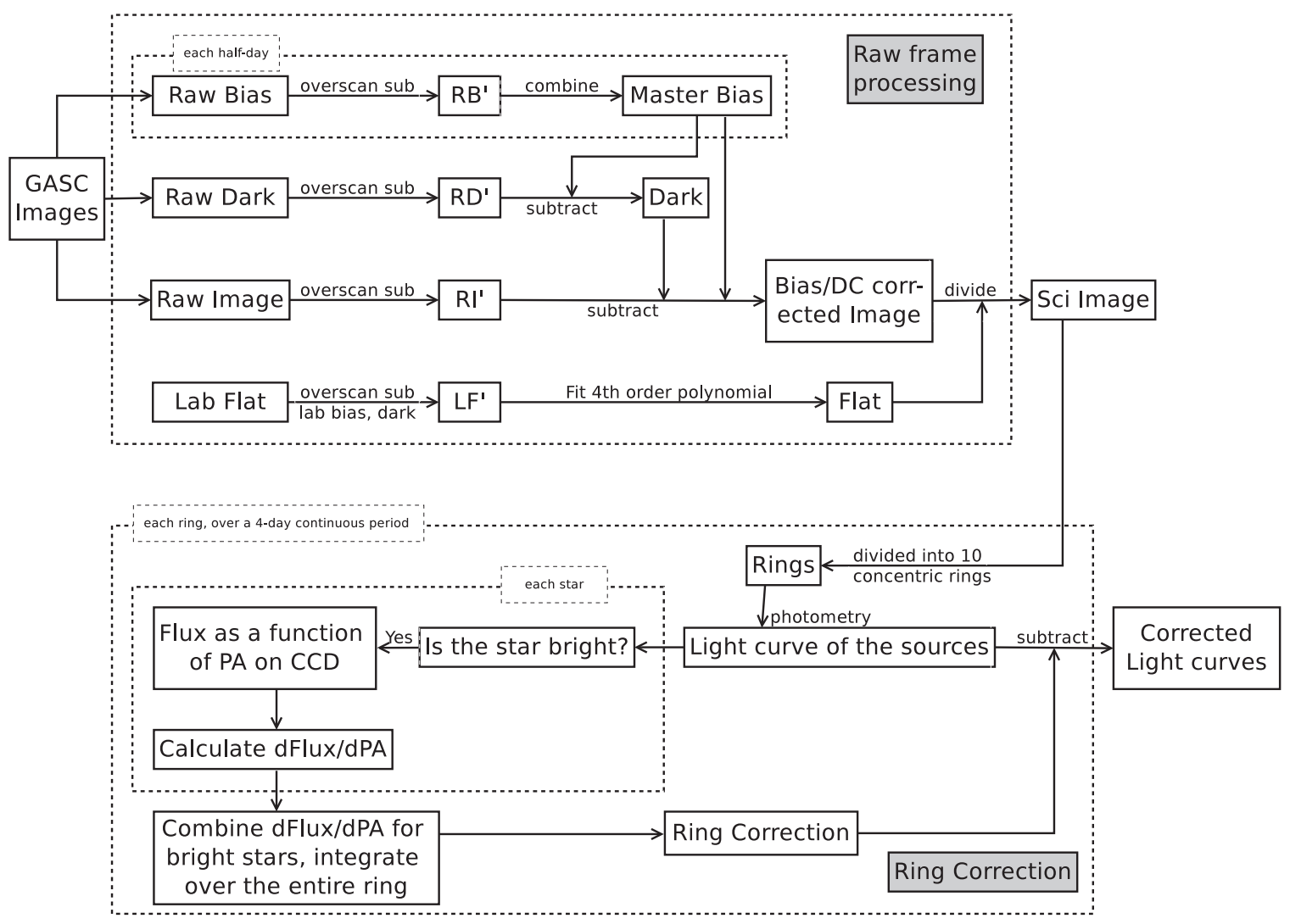

Figure 3. Flowchart showing the customized GASC data reduction pipeline.

\section{Data Reduction}

GASC has a large FOV, and the absence of a mechanical system for tracking will lead to star trails on the CCD over the course of the exposures. The instrument is fixed in orientation and stars sweep out circular arcs centered on the south pole every sidereal day. The illumination response of the GASC across the large FOV is highly variable, at times up to $30 \%$ from the center to the edge of the field, due to inherent qualities of the fisheye lens and due to mechanical baffling introduced to minimize the scattering of light due to the Moon. In addition, there are sidereal variations on the order of $0.2 \mathrm{mag}$, due to instrumental effects described in Section 3.4. A custom data reduction pipeline is comprised of a set of routines written in IDL that processes the $\sim 11,925$ raw sky frames for each filter band and produces calibrated sky brightness measurements. The pipeline by necessity also produces calibrated light curves of all the stars brighter than $\sim 7.5$ in $V$. An overview of the essential steps is presented in Figure 3. Each step is detailed in the respective subsection below.

\subsection{Pre-reduction}

The overscan region of each frame was subtracted to remove the consequences of any voltage variations. In each half-day period of observation, a "master bias frame" was made by combining single overscan-subtracted bias frames. For each half-day period of observation, this "master bias frame" has been subtracted from the data frames to remove the internal bias structure across the chip. The internal temperature variations within the heated enclosure may lead to implied (and artificial) variations of the sky brightness as well as the photometry of bright targets. We tested the possibility that the enclosure temperature and CCD temperature affect the photometric magnitudes by calculating any possible cross-correlations between the enclosure temperature and the CCD temperature, together with typical light curves for bright stars in the GASC FOV during the entire 2009 winter season. No correlations between any pairs of those factors have been identified, indicating a stable work state of GASC during the 2009 winter season and a reasonable bias subtraction technique.

Acquisition of usable sky flats for this type of system is difficult to perform on the sky, due to the non-tracking capability of the system and the sheer size of the FOV. We measured the flat-field illumination properties of GASC with a uniform illumination screen after the system was returned to Caltech from Dome A. A multiband lab flat shows that the optical center of the lens is, fortuitously, coincident with the SCP. For each photometric bandpass, a fourth-order polynomial has been directly applied to fit the lab flat. The lab flat was used as a method to remove global transmission variations across the field, whereas pixel-to-pixel variations were removed by compiling a sky reference flat. The pixel-to-pixel variations turn out to be negligible (less than $\sim 0.3 \%$ ) when compared to the photometric accuracy GASC is able to achieve. However, it was not able to remove the remaining $\lesssim 0.2$ mag variations that were removed by the "ring correction" technique, which will be discussed in Section 4.3.

\subsection{Image Profiles and Astrometry}

The DAOFIND and APPHOT packages within IRAF $^{14}$ were used to detect and perform photometry on approximately 2600

\footnotetext{
${ }^{14}$ IRAF is distributed by the National Optical Astronomy Observatories, which are operated by the Association of Universities for Research in Astronomy, Inc., under cooperative agreement with the National Science Foundation (NSF).
} 


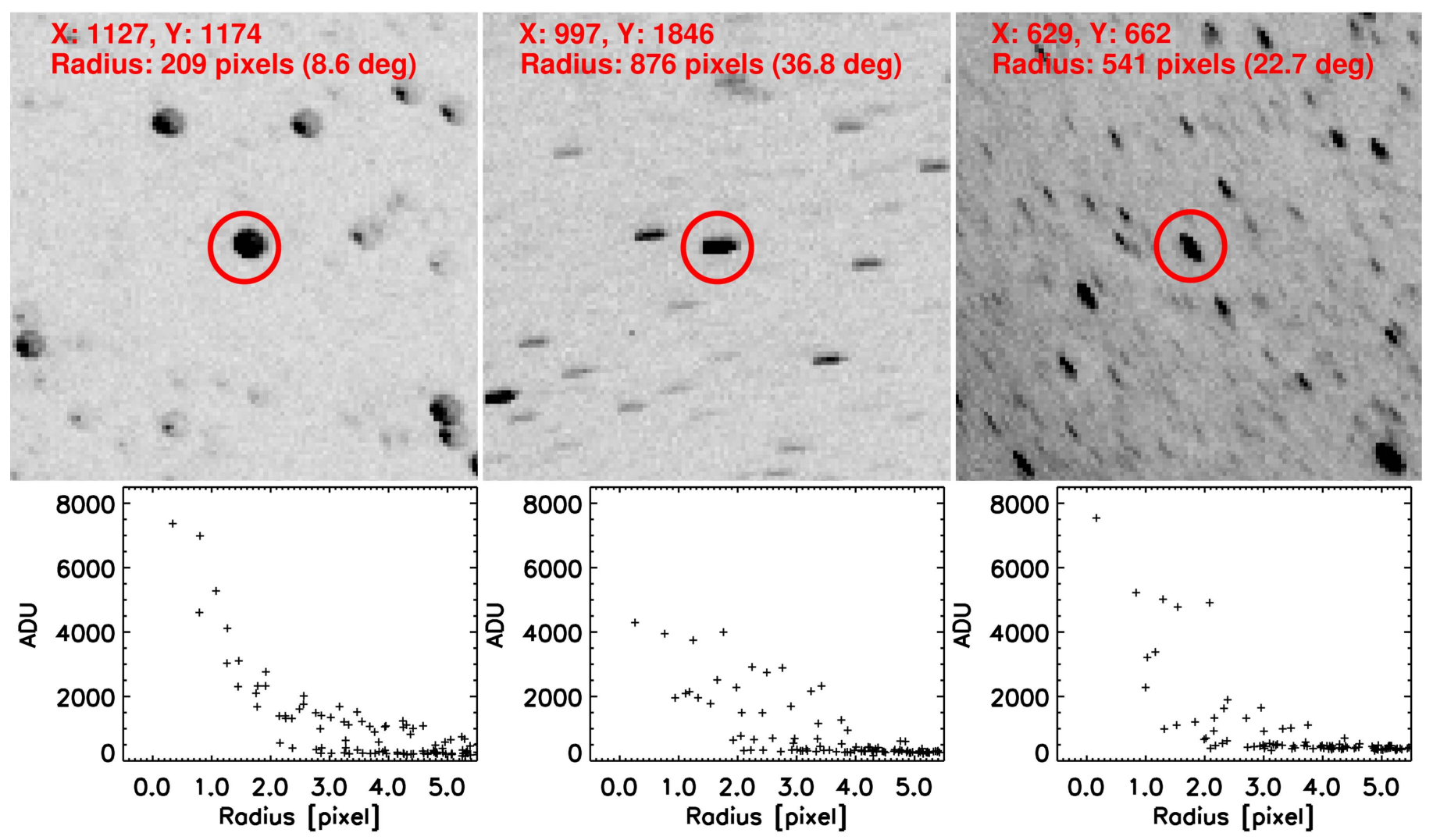

Figure 4. Typical profiles of stars at different distances to the SCP.

bright stars in the GASC FOV, most of which are between 3.5 and 7.5 mag in $V$. Without tracking, stars trail along concentric rings around the SCP and present elongated, curved PSFs on each frame. Figure 4 presents the typical profiles of stars at different distances to the SCP.

The astrometry routine adopted in the GASC data reduction pipeline makes use of the almost-polar location of the instrument. We derotated the physical coordinates of the sources in each image relative to known reference images.

To reduce the uncertainty caused by distortion and increase the accuracy of matching, as reference frames we selected 20 high-quality frames equally spaced in time over one entire rotation cycle (i.e., one sidereal day). Given the time of exposure of any other frames, all stellar coordinates can be obtained by rotating those 20 templates within $\pm 9^{\circ}$. This provided a time-economical solution for performing the astrometry required by the GASC science goals on the $\sim 36,000$ sample images. An overview of the GASC FOV and field stars used to perform aperture photometry is shown in Figure 5.

\subsection{Ring Correction}

Due to the combined effect of the presence of the cover window and the different responses of the interline transfer CCD to different incident angles, light curves for $\sim 2600$ stars imaged in the GASC FOV show asymmetrical sidereal fluctuations. The amplitude of this variation grows as the distance of stars to the SCP increases. We looked at the behavior of bright, isolated stars, which sweep out concentric rings in the GASC FOV. As the "standard stars" have higher $\mathrm{S} / \mathrm{N}$, a weighted combination of their light curves gives us feedback on the entire optical system. This feedback, however, also applies to all other stars with a lower $\mathrm{S} / \mathrm{N}$.

Here we introduce a "ring correction" to remove the residual instrumental effects, to the order of $\pm 0.2 \mathrm{mag}$ in the raw photometry. The methodology is to consider the features of the light curves for bright stars that have similar distances from the $\mathrm{SCP}$, as they sweep out paths along the same ring with different hour angles but similar decl. The systematic light curve features do not change drastically at slightly different radii. The GASC FOV has been subdivided into 10 concentric rings, each with a width of 100 pixels, with an exception of 60 pixels for the outermost ring. Figure 6 shows the concentric rings dividing the GASC FOV. Within each ring we investigated the behavior of standard stars, which are non-variable stars with $V \sim 3.5-5.5$, and mapped the gradient of its flux variation over different position angles (P.A.) on the CCD chip relative to the SCP, i.e., $d$ Flux $/ d P A$. Then, we combined the gradients calculated from each standard star at each P.A. over a continuous run of observations under good weather conditions, and applied a spline interpolation to obtain a gradient map over that ring. We then integrate over the P.A. and convert the integrated flux into magnitude. This produces a phase diagram of magnitude variations within each ring, representing the systematic behavior of the stars as they trail along certain rings of the CCD chip.

We refer to this procedure as the "ring correction." The light curve corrections for all other stars can be obtained by subtracting the "ring correction" after proper time phase matching. The "ring corrections" have been built based on a four-day continuous run of high-quality data obtained from 04:25 UT on 2009 June 22 to 03:47 UT on 2009 June 26. This has been applied successfully to the data obtained during the entire season. The "ring corrections" for typical stars within 


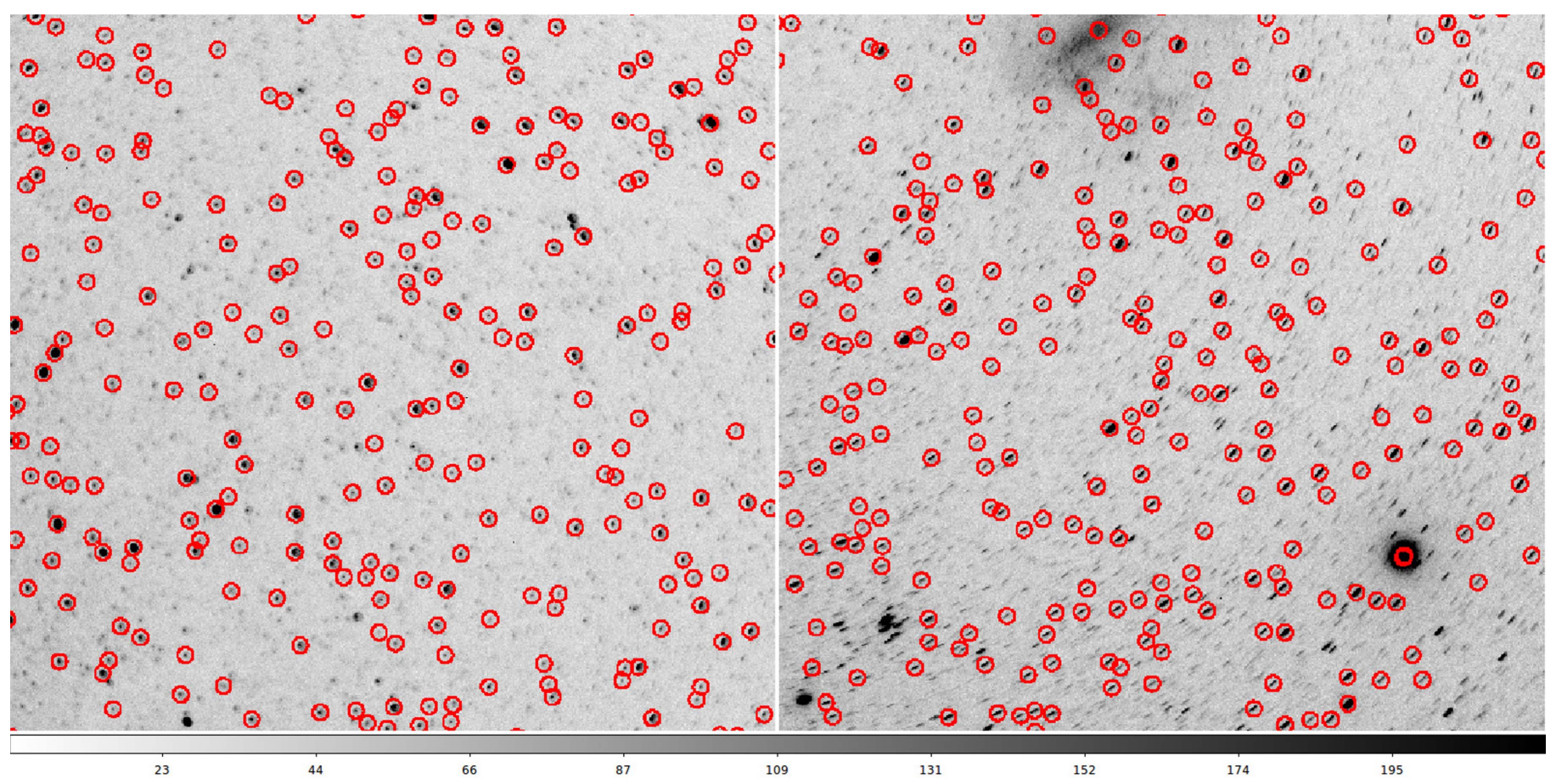

Figure 5. Stellar field in the GASC FOV obtained on 2009 June 22. The left panel shows the central FOV and the right panel shows one corner of the FOV. Sources chosen to perform aperture photometry have been circled by $r=4$ pixel apertures. The images were taken in defocused mode to account for the huge pixel scale. The right panel shows significant star tracks near the corner of the FOV due to the Earth's rotation.

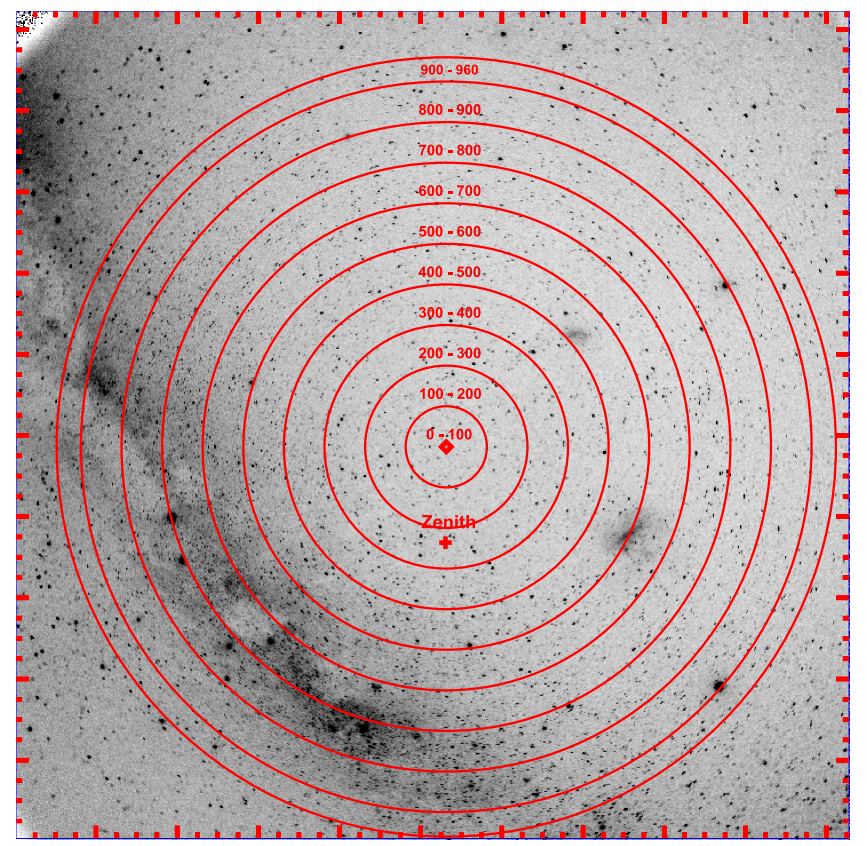

Figure 6. Concentric rings dividing the GASC FOV. The + marks the physical position of the zenith on the GASC FOV.

each of the 10 rings are shown in Figure 7, and they work well for most of the cases. Additionally, the $\sigma$-magnitude diagram, after applying both the pseudo-star correction and the ring corrections, is shown in Figure 8. For instance, we obtained $\sim 3 \%$ photometric accuracy for stars with apparent magnitude $V \approx 5.5$. In summary, with the ring correction procedure completed, the light curves have been corrected for instrumental effects that cause intensity variations across the field and as a function of time.

\subsection{Calibration for Sky Brightness}

\subsubsection{Determination of Catalog Magnitude}

This step of the GASC data reduction pipeline converts instrumental magnitudes to catalog magnitudes. Furthermore, the sky brightness can be determined by applying this offset to the GASC-measured sky flux. If we define the "radius" of each star as its distance from the SCP (pixel coordinates $X=1063$, $Y=972$ ), we find that the amplitude of the daily fluctuation in a star's light curve depends on (1) its radius, (2) observing bandpass, as shown in Figure 9, together with (3) the mean value of the difference between the standard star's catalog and instrumental magnitude. As the radius increases, the more stable is the mean difference between a standard star's catalog and instrumental magnitude, and the less affected is the standard star's flux is due to instrumental effects.

The upper panel in Figure 9 shows a stable trend of the mean difference between the standard stars' catalog and instrumental magnitudes in the $V$ - and $R$-bands, which means that as stars travel around in the GASC FOV, though the distance to the SCP varies for different stars, it is still reasonable to treat the brightest magnitude in one cycle as the true instrumental magnitude of that star. Instrumental effects become more significant near the edges of the FOV. Strong geometrical distortions, as well as the large incident angle near the edges of the FOV, will cause an unexpected and non-negligible reduction of the flux transmitted through the optical system. Giving special consideration to the case of the $B$ - and $V$-bands, we set a cutoff radius of 700 pixels, corresponding to $\sim 30^{\circ}$ from the SCP, and we use all of the standard stars within this radius to calibrate the sky brightness.

We rely on the linearity of the CCD and minimize the $\chi^{2}$ value of the fit using the offset between the standard stars' photometric magnitudes and their catalog magnitudes. We 

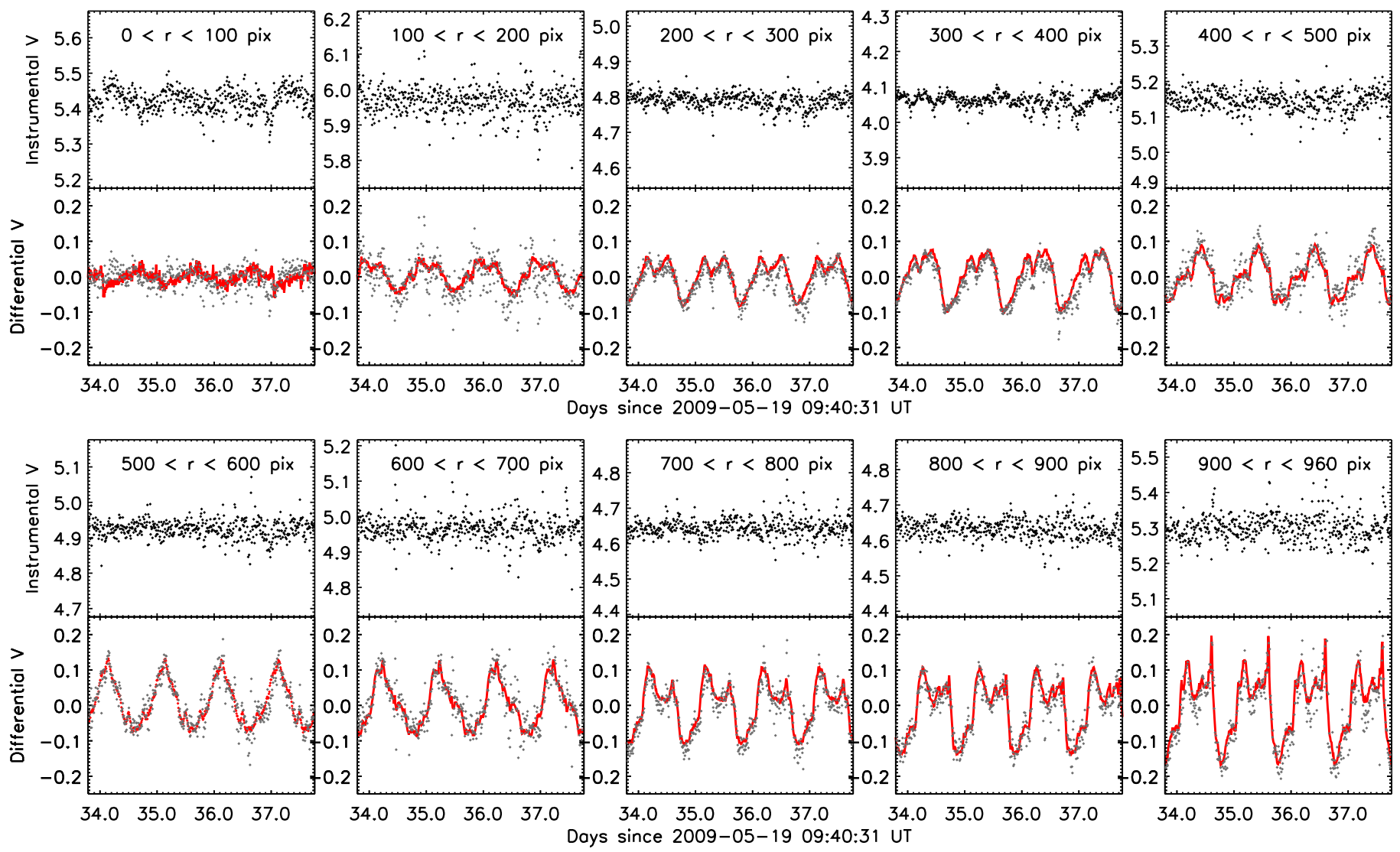

Figure 7. "Ring corrections" for $V$-band light curves for 10 different annuli are shown as 10 subfigures. Each panel represents an annulus width of 100 pixels in radius. The upper subpanels represent the output light curves after applying the ring corrections. In the lower subpanels, the gray dots represent the input light curves before applying the corrections, and the red symbols represent the models of corrections within each corresponding radius range.

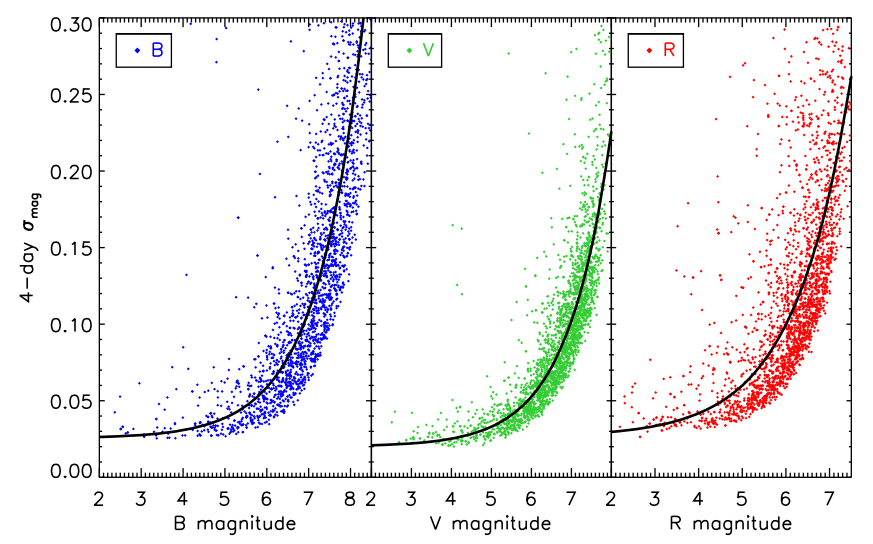

Figure 8. Photometric errors vs. stellar brightness. From left to right, we show the photometric accuracy in the Bessell $B-, V-, R$-bands, respectively, after applying the "ring corrections." The photometric uncertainties were calculated from data obtained on four consecutive days.

consider data only within 700 pixels of the SCP and weight by the area of each ring. This gives us our multiband sky brightness measures at Dome A calibrated by the standard stars. Once GASC was shipped back to Caltech, we performed tests at Palomar Observatory. Table 1 gives the $B V R$ photometric offsets from instrumental to calibrated values. The details of the Palomar GASC test are discussed in the next section. The offset in the constant term between the Palomar and Dome A calibration model was due to the absence of the cover window in the Palomar test and the different exposure times between two observation epochs.

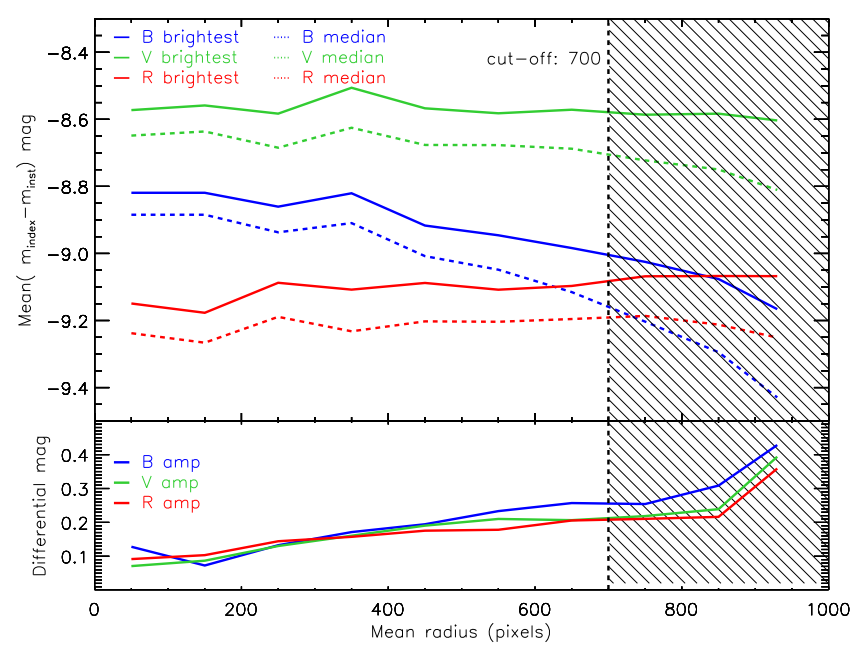

Figure 9. Radius-magnitude offset diagram for the "ring correction" for different radii. The offset between the standard stars' catalog magnitude and instrumental magnitude has been calculated based on two different considerations of instrumental magnitude. The results are based on the median values of all of the standard stars' brightest (represented by solid lines) and median (represented by dashed lines) magnitudes during a sidereal day. The lower panel shows the radius-amplitude diagrams for the "ring correction" in different annuli. A significant increase in amplitude occurs if the radius is increased from 700 to 800 pixels. A vertical dashed line and the shaded region indicate the 700 pixel radius cutoff for stars to be used for calibration.

\subsubsection{Determination of Photometric Conditions}

Variations of global transparency, including weather changes, possibly snow and frost that formed in front of the 
Table 1

Calibration Models

\begin{tabular}{llll}
\hline \hline Band & Dome A Median mag & Dome A Brightest mag & Palomar \\
\hline$B$ & $m_{\text {inst }}-9.02$ & $m_{\text {inst }}-8.92$ & $m_{\text {inst }}-9.52$ \\
$V$ & $m_{\text {inst }}-8.67$ & $m_{\text {inst }}-8.56$ & $m_{\text {inst }}-9.00$ \\
$R$ & $m_{\text {inst }}-9.21$ & $m_{\text {inst }}-9.10$ & \\
\hline
\end{tabular}

enclosure's cover window, will dramatically affect many quantities in measuring sky brightness, the fraction of the sky covered by clouds, as well as the photometry of bright sources. This global effect can be subtracted off by introducing a "pseudo-star" with a count rate $f^{p}$ and instrumental magnitude $m^{p}=-2.5 \times \log _{10} f^{p}$, which has been constructed from the observed counts of 2600 target stars in each frame according to

$$
f_{i}^{p}=\sum \frac{f_{i, j}}{\left(\sigma_{j}^{\text {ring }}\right)^{2}+\left(\sigma_{i, j}\right)^{2}}, \quad m^{p}=-2.5 \times \log _{10} f^{p}+\mathrm{ZP}^{P},
$$

where $i$ is the frame number in the observing sequence and $j$ is the star number in each frame. $\sigma_{j}^{\text {ring }}$ gives the standard deviation of the residuals in counts for the $j$ th star after the ring correction during the four-day continuous run of highquality data obtained from 04:25 UT on June 22 to 03:47 UT on June $26, \sigma_{i, j}$ gives the measured photometric error for the $j$ th star in the $i$ th observation, and $\mathrm{ZP}^{P}$ is the zero point for instrumental magnitude and assigned to be 25 . We subtract $m^{p}$ from the rough photometric results to remove the global variations in the entire GASC FOV. Furthermore, the variation of the pseudo-star can be an indicator of transparency variations and further used to estimate the cloud coverage. A more detailed discussion will be presented in the following sections.

\subsubsection{GASC Test at Palomar Observatory}

To test the quality of GASC measurements and the calibration of sky brightness, another experiment intended to measure and calibrate the sky brightness at an astronomical site was implemented at Palomar Mountain Observatory. The sky at Palomar during a moonless night is sufficiently dark to check the Dome A measurements. The Palomar Night Sky Brightness Monitor (NSBM) ${ }^{15}$ allows a real-time comparison between the night sky brightness measured by the two different instruments. The Palomar NSBM consists of two units deployed at Palomar Observatory. A remote photometer head and a base station receive data from the remote head via a wireless spreadspectrum transceiver pair. The remote head has two photometers that sample areas of the sky $\sim 5^{\circ} .6$ in diameter at two elevation angles. The photodetectors used to measure the sky brightness receive filtered light to define a spectral response centered in the visual range, with a strong cutoff in the nearinfrared.

One unit of the NSBM uses a $1.5 \mathrm{~cm}$ diameter photodetector, which measures the brightness of the sky $\sim 5^{\circ} .6$ in diameter at the zenith. Without rejecting stellar contaminants, the mean

\footnotetext{
$\overline{15}$ http://www.sao.arizona.edu/FLWO/SBM/SBMreport_McKenna_ Apr08.pdf
}

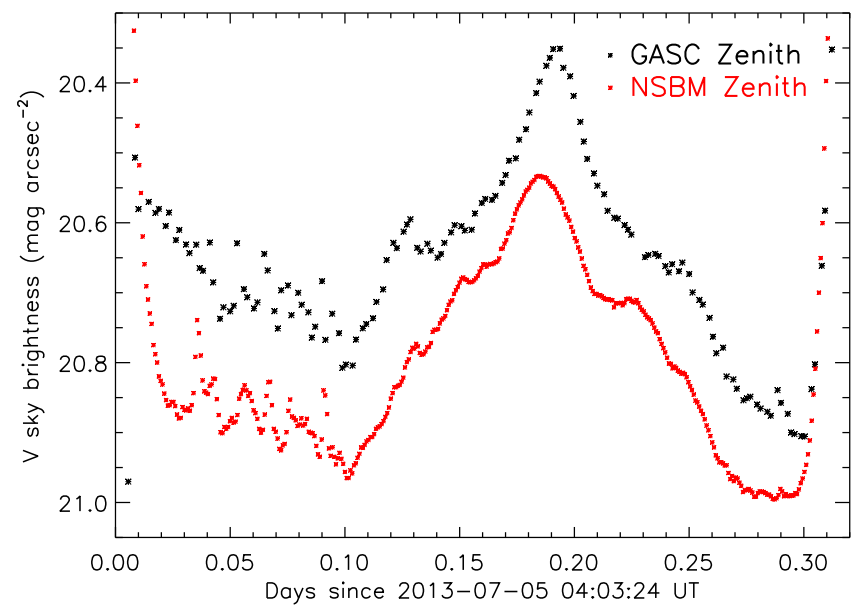

Figure 10. Palomar night sky brightness measured and calibrated by NSBM (red dots) and GASC (black dots) on UT 2013 July 05.

value for this region is taken to represent the night sky brightness. The output data from the NSBM consists of the measured frequency and ambient temperature of each sensor. The sky brightness is calculated as ${ }^{16}$

$$
\begin{aligned}
\text { Zenith magnitude }= & -2.5 \log _{10}(\text { Zenith reading }-0.012) \\
& +\mathrm{ZP} .
\end{aligned}
$$

The detector output frequency (in Hertz) constitutes the raw data, as the NSBM uses a light to frequency converter. The dark frequency to be subtracted for the zenith is $0.012 \mathrm{~Hz}$. The zero point of the NSBM system adjusted to the National Parks System from one night's data (2013 July 4) is $19.41 \mathrm{mag} \mathrm{arcsec}^{-2}$, and for a band comparable to the Johnson $V$-band is 18.89 . Figure 10 shows the time variations of the sky brightness measured by NSBM and GASC. The sky brightness measured by two different instruments, with two completely different calibration methods, agrees overall to $\sim 0.12$ mag $\operatorname{arcsec}^{-2}$.

A separate test was conducted at Palomar to show that the camera orientation, specifically the azimuth angle of the camera, results in a variation in the magnitudes of bright stars. This test used exposures taken very close to one another in time. The results of this test confirmed the variations we see in the original data.

\section{Results and Discussion}

\subsection{Sources of Sky Brightness}

Artificial light pollution is essentially nonexistent at Dome A Antarctica. The main contribution to the sky background is usually from the atmospheric scattering of the light from the Sun and the Moon. At Dome A $\left(80^{\circ} 22^{\prime} \mathrm{S}, 77^{\circ} 21^{\prime} \mathrm{E}\right)$, there is some twilight time even on the first day of the southern winter, as the Sun is roughly $13^{\circ} .8$ below the horizon at local noontime. The closer the Sun is to the horizon at local noontime on other days of the year, the greater will be the variation of the sky brightness, even on days when the Sun does not rise and set.

Airglow persistently provides photon emission and gives the dominant component of the optical and near-IR night sky

\footnotetext{
16 Zenith readings are available at http://bianca.palomar.caltech.edu/ maintenance/darksky/index.tcl
} 
brightness (Benn \& Ellison 1998). The Antarctic sites such as Dome A, however, are particularly prone to aurorae that can be extremely bright in the optical passbands. Broadband filters and low resolution spectrographs covering the auroral lines are sufficiently likely to be contaminated by strong emission lines from aurorae, i.e., the $\mathrm{N}_{2}$ second positive (2P) and $\mathrm{N}_{2}^{+}$first negative $(1 \mathrm{~N})$ bands dominating the $U$ and $B$ bands, the [OI] $557.7 \mathrm{~nm}$ emission dominating the $V$-band, and the $\mathrm{N}_{2}$ first positive (1P), $\mathrm{N}_{2}^{+}$Meinel $(\mathrm{M})$, and $\mathrm{O}_{2}$ atmospheric bands dominating the $R$ and $I$ bands (Gattinger \& Jones 1974; Jones \& Gattinger 1975). Customized filters or spectrographs with a moderately high resolving power can minimize the contamination from aurora and airglow emissions. We refer to Sims et al. (2012b) for a more comprehensive review of airglow and aurorae as dominant sources of sky brightness in Antarctica sites.

Diffuse light from the Milky Way Galaxy could also contribute to the sky brightness. The Galactic latitude $b$ of the SCP is $-27^{\circ} .4$, and part of the Galactic plane was included in the GASC FOV. The plate scale of GASC is approximately $147^{\prime \prime}$ per pixel, and the subpixel stellar contamination needs to be calculated and removed from the measured sky brightness data. Airglow, zodiacal light, and aurorae also contribute to the sky brightness. The intensity and frequency of the occurrence of aurorae depend upon the solar activity. Rayleigh (1928) and Rayleigh \& Jones (1935) were the first to note a correlation between the sky brightness and the 11 year solar cycle. This is due to the airglow being brighter at solar maximum and fainter at solar minimum (Krisciunas 1997; Krisciunas et al. 2007). The $10.7 \mathrm{~cm}$ radio flux of the Sun is widely used as an index of solar activity. The 2009 winter season occurred during solar minimum, so the sky at Dome A should have been as dark as other sites at solar minimum, or $B \approx 22.8 \mathrm{mag} \mathrm{arcsec}^{-2}$ and $V \approx 21.8 \mathrm{mag} \operatorname{arcsec}^{-2}$. We do not expect that the Dome A measurements of 2009 are significantly affected by auroral events.

An approach to determine the sky brightness and estimate the cloud cover is given in the following subsections. Due to the extremely wide FOV and the fisheye optical design of GASC, scattered light from the edges of the optical system, as well as reflection and refraction inside the optical system, is inevitable. The actual contribution from the Sun and the Moon cannot be well modeled when the sky becomes too bright. A rough model of the Sun and the Moon's contribution to the sky brightness will be discussed.

\subsection{GASC Measurements of Sky Brightness}

The sky brightness is transformed from analog-to-digital units (ADU) into units of mag $\operatorname{arcsec}^{-2}$ for each photometric band. The GASC instrumental magnitude is defined as

$$
m_{0}=25-2.5 \log _{10}(\mathrm{ADU}) \text {. }
$$

The sky brightness in units of mag $\operatorname{arcsec}^{-2}$, which varies from band to band, can be defined as

$$
S_{\lambda}=a+b \times\left[25-2.5 \times \log _{10}\left(\mathrm{ADU} / \mathrm{pix}^{2}\right)\right]
$$

where "pix" is the pixel scale in units of arcsec pixel ${ }^{-1}$. The constant term in linear calibration models is $a$, and the coefficient scaling the instrumental magnitude is $b$. In a certain sky region we wish to calibrate, we draw a box and investigate the statistics of the ADU values among all the pixels inside. We choose the "mode" value to best represent the sky brightness, which is a more stable measurement as it is less affected by contamination from the bright sources, the widespread PSF of stars due to the GASC optical system, and other unexpected events such as bright local aurorae. However, even the smallest pixel scales in GASC are $147.3 \operatorname{arcsec}$ pix $^{-1}$ near the center of the FOV, corresponding to a box of $2.5 \times 2.5$ on the sky. The measured sky brightness will inevitably be contaminated by the unresolved faint sources.

We looked at several small regions that lack bright sources to reduce the effect of stellar contamination. For instance, a box centered at R.A. $=2^{\mathrm{h}} 24^{\mathrm{m}}$, decl. $=-86^{\circ} 25^{\prime}$ and $25 \times 25$ pixels in size $\left(\sim 1^{\circ} \times 1^{\circ}\right)$ was inspected. The $B$-band and $R$-band magnitudes of 9550 stars in this region were obtained from the USNO-A2.0 catalog. We estimated a stellar contamination of $24.14 \mathrm{mag} \mathrm{arcsec}^{-2}$ in the $B$-band. Using a mean $V$-band contamination of $23.31 \mathrm{mag} \operatorname{arcsec}^{-2}$ and a calculated median color of $V-R=0.4$ mag based on the catalog from Landolt (1992), we estimated the $R$-band contamination to be $22.91 \mathrm{mag} \operatorname{arcsec}^{-2}$.

Figure 11 shows the sky brightness variations during the 2009 observing season. At such a southerly latitude as that of Dome A, the Moon is always fairly full when it is above the horizon from April to August, leading to a strong correlation between lunar elevation and sky brightness (Zou et al. 2010). There is a monthly variation of sky brightness, which is strongly correlated with the lunar elevation angle. The GASC sensitivity did not allow data acquisition when the sky brightness was above a certain threshold. A dramatic enhancement in the sky brightness can be identified by looking at the data obtained late in the 2009 winter season. Figure 12 is a zoomed-in plot for four consecutive days during the midwinter of 2009. In Figure 13, the Moon's contribution is negligible when it is more than $7^{\circ}$ below the horizon. However, a variation of the sky brightness of more than 1 mag $\operatorname{arcsec}^{-2}$ can be identified, which shows a strong correlation with the Sun's elevation angle.

\subsection{Comparison with Sky Brightness at Palomar}

Additional tests of GASC were conducted at Palomar Observatory. ${ }^{17}$ The "ring correction" to light curves and the fitting of calibration models only work based on an entire cycle of the track of the stars. This allows the determination of the position within a ring where stars are least affected by instrumental effects. Though it is not feasible to find the maximum transmission for each star cycle from tests at Palomar, we can still point GASC near the zenith and obtain different calibrations based on the instrumental magnitudes measured by GASC and the corresponding catalog magnitudes.

On 2013 July 5, GASC arrived at Palomar Observatory and was reassembled. Two tests were carried out. The first test was to compare GASC-measured sky brightness with Palomar NSBM measurements. We pointed the GASC at the zenith and set the exposure time to $50 \mathrm{~s}$ for the Bessell $B, V$, and $R$ filters. ${ }^{18}$ The calibration was carried out based on single frames of high image quality for each of the bandpasses. We used the instrumental magnitudes of the standard stars in one single high-quality frame per filter taken under photometric conditions. This is different than

\footnotetext{
${ }^{17}$ Geographical coordinates of Palomar Observatory: latitude $33^{\circ} 21^{\prime} 21^{\prime \prime} \mathrm{N}$, longitude $116^{\circ} 51^{\prime} 50^{\prime \prime} \mathrm{W}$.

18 For the measurements at Palomar, we note a roughly $0.5 \mathrm{mag} \operatorname{arcsec}^{-2}$ variation of the sky brightness over the course of the night due to the band of the Milky Way passing overhead.
} 


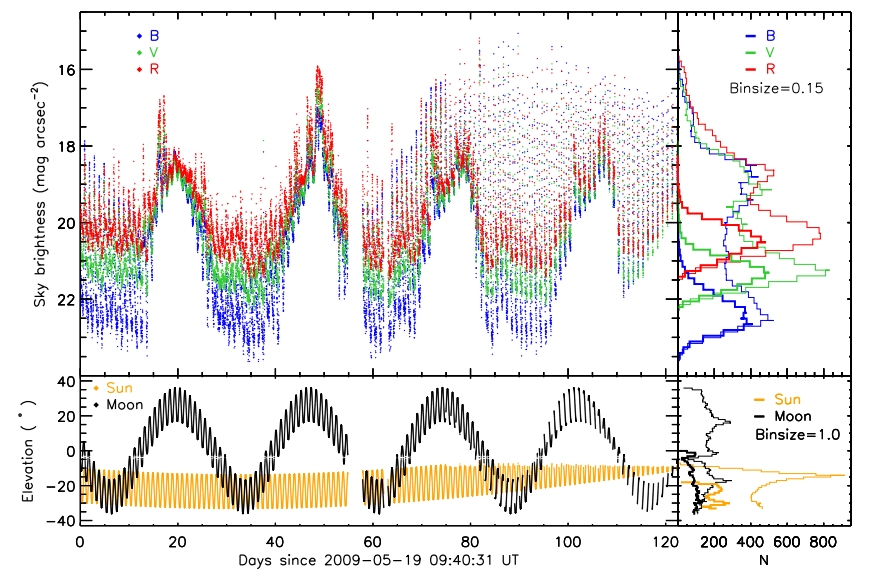

Figure 11. Multiband sky brightness within a 1 square degree region near the SCP, as well as the Sun's and Moon's elevation during the 2009 winter season. The upper- and lower-left panels present the time series while the top and bottom right-hand panels show the histograms. The results for the Bessell $B$-, $V$-, and $R$-bands are represented by blue, green, and red symbols, respectively. In the right panels, the histograms with solid thick lines represent the statistics for sky brightness during dark time, when the solar elevation angle is less than $-18^{\circ}$ and the lunar elevation angle is less than $0^{\circ}$. Stellar contamination has already been removed by subtracting the contribution of a total of 9550 stars in the inspection area. Their magnitudes were obtained from the USNO A-2.0 catalog.

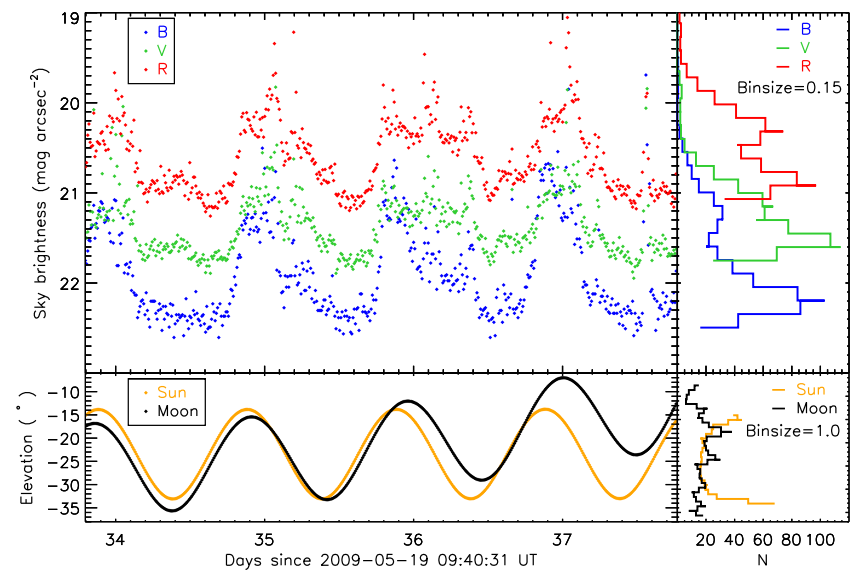

Figure 12. Four-day subset of data shown in Figure 11, from 04:25 UT on 2009 June 22 through 03:47 UT on 2009 June 26. When the Moon is many degrees below the horizon, the daily variation of sky brightness is dominated by the elevation of the Sun.

the method used for data obtained at Dome A, where the brightest instrumental magnitudes over the course of a day were adopted as the throughput of the system.

For each star in the FOV of each single exposure, the orientation of its maximum transmit position on the CCD chip is randomly distributed. In order to compare the Palomar calibration with the calibration of Dome A data (whose calibration models have been based on the standard stars' maximum transmitted flux), we performed another calibration of Dome A data, based on the median instrumental magnitude of each standard star as it tracks during one daily cycle to simulate the calibration that use the stars' flux at random positions like the Palomar test. By treating either the brightest or the median magnitude of standard stars along complete circles in Dome A data as the instrumental magnitude, an intrinsic offset of $(-8.670)-(-8.564)=-0.11$ magnitude is obtained due to the different measures of instrumental magnitude. The difference in the $V$-band median

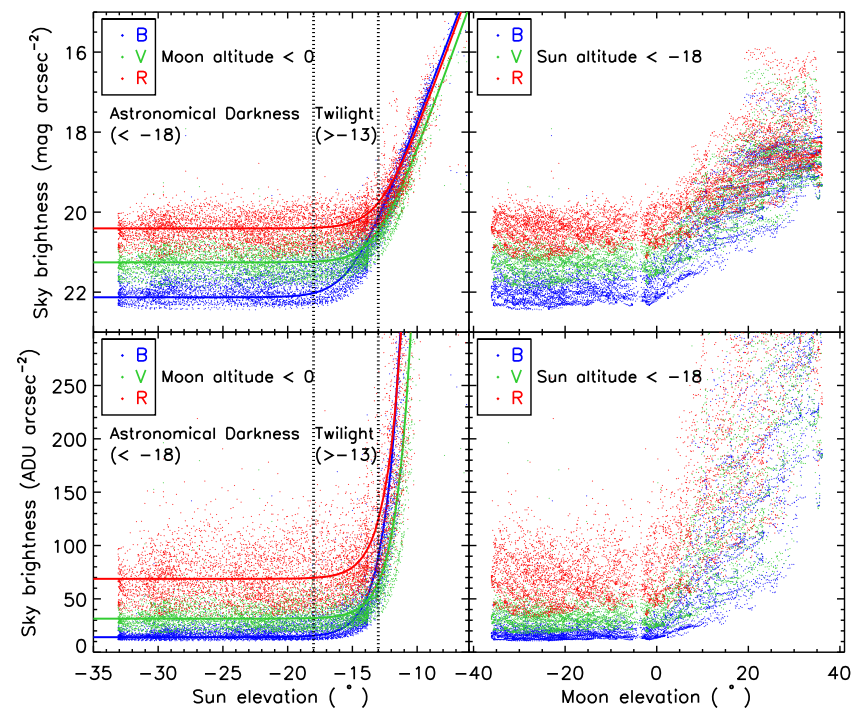

Figure 13. Multiband sky brightness vs. the Sun and Moon elevation. The upper panels show the measurements in mag $\operatorname{arcsec}^{-2}$ while the lower panels show the data as ADUs per square arcsec. The left-hand panels show the relation between the sky brightness and the elevation angle of the Sun together with the model from Equation (7). Only the data with Moon elevation less than $0^{\circ}$ have been included. The right panels show the relation between the sky brightness and the elevation of the Moon. Only the data with Sun elevation less than $-18^{\circ}$ have been included.

sky brightness on the night of 2013 July 5 UT at Palomar Observatory, as measured by NSBM and GASC, was $(20.880-20.653)=0.23 \mathrm{mag} \operatorname{arcsec}^{-2}$. Thus, GASC and NSBM agree within $-0.11+0.23=0.12 \mathrm{mag} \mathrm{arcsec}^{-2}$, and the "ring calibration" method gives a reasonable calibration for the GASC data.

Usually, inland astronomical sites are affected to some degree by artificial light pollution from populous cities. The sky brightness as a function of elevation angle obtained from the Tucson lab sites shows that there is a significant difference in sky brightness between the zenith and $20^{\circ}$ elevation (McKenna 2008). At Cerro Tololo Inter-American Observatory, the $V$-band sky brightness deviates from the model of Garstang (1991) due to light pollution at elevation angles of $\lesssim 10^{\circ}$ in the direction of La Serena (Krisciunas et al. 2010). Without accounting for stellar contamination, Table 2 presents the median sky brightness for different regions at Dome A Antarctica during the 2009 winter season, both for the dark time and whole season (the values within parentheses). Five concentric circular areas, of increasing radius and centered at the SCP, were inspected. Though the regions were centered at the SCP instead of the zenith, the approximate $10^{\circ}$ offset has been ignored. From Table 3, no significant increase in brightness can be identified as a function of increasing angular radius. This indicates that within $30^{\circ}$ of the SCP there is dark sky that remains roughly constant in brightness.

\subsection{Sun and Moon Model}

Liu et al. (2003) modeled the relationship between the sky brightness and the phase and elevation angle of the Moon. Independent of the scattering of light caused by reflection and refraction in the GASC optical system, the $B-, V$-, and $R$-band data should exhibit the same functional form relating to the 
Table 2

Sky Brightness for Different Percentages of Time Value ${ }^{\mathrm{a}}$

\begin{tabular}{|c|c|c|c|c|c|c|}
\hline Band & Value $^{\mathrm{b}}$ & $80 \%$ & $50 \%$ & $20 \%$ & $10 \%$ & $5 \%$ \\
\hline \multirow{3}{*}{$B$} & Mode & 21.68 (19.17) & $21.99(20.91)$ & $22.22(21.95)$ & $22.31(22.15)$ & 22.37 (22.26) \\
\hline & Subtracted & $22.01(19.20)$ & $22.45(21.06)$ & $22.82(22.40)$ & $22.98(22.70)$ & $23.10(22.90)$ \\
\hline & Corrected & $22.13(19.32)$ & 22.57 (21.18) & $22.94(22.52)$ & $23.10(22.83)$ & $23.22(23.02)$ \\
\hline \multirow{3}{*}{$V$} & Mode & $20.93(19.05)$ & $21.22(20.61)$ & $21.48(21.24)$ & $21.59(21.43)$ & $21.67(21.56)$ \\
\hline & Subtracted & $21.07(19.08)$ & $21.40(20.70)$ & $21.72(21.42)$ & $21.86(21.65)$ & $21.96(21.81)$ \\
\hline & Corrected & $21.19(19.20)$ & $21.52(20.83)$ & $21.84(21.54)$ & $21.98(21.77)$ & $22.08(21.93)$ \\
\hline \multirow{3}{*}{$R$} & Mode & 20.13 (18.69) & $20.44(19.91)$ & 20.75 (20.49) & $20.90(20.70)$ & $20.99(20.85)$ \\
\hline & Subtracted & $20.21(18.71)$ & 20.56 (19.98) & $20.91(20.61)$ & $21.68(20.85)$ & $21.20(21.03)$ \\
\hline & Corrected & $20.34(18.54)$ & $20.68(20.10)$ & $21.03(20.73)$ & $21.02(20.97)$ & $21.15(21.32)$ \\
\hline
\end{tabular}

Notes.

a Values without parentheses are for dark time. Values in parentheses are for the whole season.

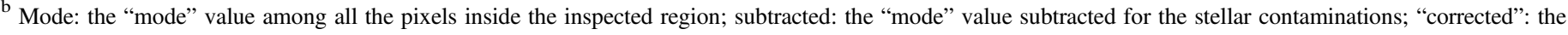
"subtracted" values further corrected for the offset between the GASC and Palomar NSBM.

Sun's and Moon's effects. We can write

$$
F_{\text {Sun }}=a 10^{b \theta}+c,
$$

where $F_{\text {Sun }}$ gives the sky flux when the Moon's contribution is negligible, and $a, b$, and $c$ are constants determined for different bandpasses and $\theta$ is the elevation angle of the Sun. The multiband sky brightness has been fitted with a nonlinear least-squares method using the images with good transparency and negligible contributions from the Moon.

The model for the sky surface brightness due to the Moon's contribution involves factors such as the Earth-Moon distance and the Moon's phase. Following Liu et al. (2003), the apparent magnitude of the Moon can be approximated by this empirical formula:

$$
V(R, \Phi)=0.23+5 \log _{10} R-2.5 \log _{10} P(\Phi),
$$

where $R$ is the Earth-Moon distance in astronomical units, $\Phi$ is the lunar phase angle, and $P(\Phi)$ is the function of the full Moon luminance. Following Zou et al. (2010), we apply the same approach to the sky surface brightness contribution by the Moon. $F_{\text {Moon }}$ can be expressed as a form of Equation (7) multiplied by the Moon phase factor $P(\Phi)$. Then,

$$
F_{\text {Moon }}=A P(\Phi) 10^{B \Theta}+C,
$$

where $\Theta$ is the elevation angle of the Moon and $A, B, C$ are constants determined for each bandpass. For a more refined but slightly complicated sky brightness model, one can consult Krisciunas \& Schaefer (1991). The multiband sky brightness has been fitted with a nonlinear least-squares method using images with good transparency and negligible contribution from the Sun. The models for the Sun's and the Moon's effect are shown in Table 4.

\subsection{Astronomical Twilight}

When the Sun sets, civil twilight occurs, by definition, when the Sun is $12^{\circ}$ below the horizon. Astronomical twilight ends when the Sun reaches $18^{\circ}$ below the horizon. If the sky brightness changes when the Sun is farther below the horizon, it is due to changes in the airglow contribution, aurorae, or stellar contamination. However, the definition of twilight depends not only on the photometric bandpass, but also on the atmospheric conditions at the site. Figure 13 shows the
Table 3

Mode of Sky Brightness for Regions of Different Angular Sizes ${ }^{\mathrm{a}}$

\begin{tabular}{lccc}
\hline \hline Diameter (deg) & $B$ & $V$ & $R$ \\
\hline 4.6 & $21.92(20.41)$ & $21.16(20.25)$ & $20.40(19.65)$ \\
20 & $21.90(20.40)$ & $21.16(20.27)$ & $20.39(19.66)$ \\
40 & $21.90(20.41)$ & $21.17(20.30)$ & $20.40(19.69)$ \\
60 & $21.96(20.46)$ & $21.24(20.37)$ & $20.47(19.77)$ \\
\hline
\end{tabular}

Note.

${ }^{\text {a }}$ Values without parentheses are for dark time. Values in parentheses are for the whole season.

relationship between the Sun and the Moon elevation on the sky brightness. The flux from the Moon, however, becomes significant only very close to the time of moonrise. Table 5 roughly shows the quantitative effect of the Sun's elevation below the horizon on the sky brightness.

Figure 14 shows the measured sky brightness in $B, V$, and $R$ (the top panel). The middle panel shows our model of the solar and lunar contributions to the sky brightness. The bottom panel shows the observed sky brightness minus the contributions of the Sun and Moon from our model. The residuals are predictably flatter because we have subtracted off the contribution of the Moon when it is above the horizon. Theoretically, the contributions of the aurora and airglow can be estimated after properly removing the solar and lunar contributions to the sky background. However, there is still a significant fraction of scattered light that cannot be well modeled within the area of study $20^{\circ}$ in diameter, especially when the Moon has a higher elevation angle. Hence, we do not provide any quantitative estimate of aurora and airglow in our inspecting area. During the 2009 observing season there were few large enhancements of the sky brightness when the Sun and Moon had low elevation angles. We have minimal evidence of aurorae in our data.

\subsection{Extinction, Transparency Variations, and the Estimation of Cloud Cover}

The GASC FOV was centered near the SCP and extended to a zenith angle of $40^{\circ}$. The "air mass" $X$ is the path length through the atmosphere at zenith angle $z$ compared to the path length at the zenith, and $X=\sec (z)$. At $z=40^{\circ}, X \approx 1.3$. At 
Table 4

Sun and Moon Models for Sky Brightness

\begin{tabular}{lcc}
\hline \hline Band & Sun Model & Moon Model \\
\hline$B$ & $F_{\text {Sun }}=2.076 \times 10^{6} \times 10^{0.342 \theta}+16.283$ & $F_{\text {Moon }}=118.098 \times P(\Phi) 10^{0.017 \Theta}-18.544$ \\
$V$ & $F_{\text {Sun }}=1.596 \times 10^{6} \times 10^{0.360 \theta}+35.463$ & $F_{\text {Moon }}=151.629 \times P(\Phi) 10^{0.015 \Theta}-26.084$ \\
$R$ & $F_{\text {Sun }}=2.158 \times 10^{6} \times 10^{0.353 \theta}+75.622$ & $F_{\text {Moon }}=232.785 \times P(\Phi) 10^{0.013 \Theta}-31.993$
\end{tabular}

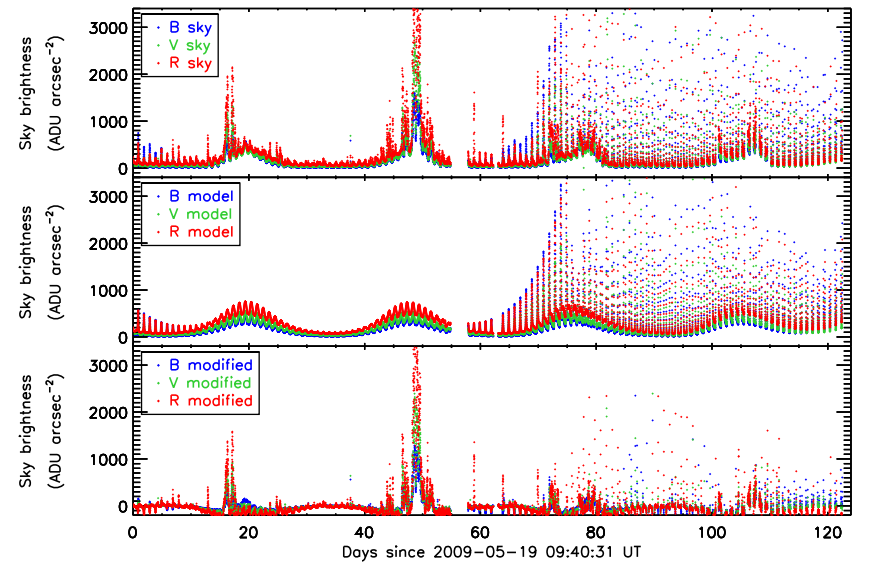

Figure 14. Application of the sky brightness models to correct the effects of the Sun and the Moon. Top panel: measured sky brightness in ADUs per square arcsec. Middle panel: our Sun and Moon model in the same units. Bottom panel: data from the top panel minus the Sun and Moon model shown in the middle panel.

the far south latitude of Dome A, any individual star within $40^{\circ}$ of the zenith exhibits a small range of zenith angle over the course of the night. GASC observed many stars at any given time over a range of 0.3 air masses. Moreover, the measurement of atmospheric extinction with GASC data is made more complicated by vignetting, the angular response of the interline sensor, as well as the different paths of light transmission through the cover window.

Atmospheric extinction is expected to be small at Dome A. For reference, at the summit of Maunakea, Hawaii (which has a comparable elevation of $4205 \mathrm{~m}$ ), the mean $B$ - and $V$-band extinction values are 0.20 and 0.12 mag airmass $^{-1}$, respectively (Krisciunas et al. 1987). The $R$-band extinction would be lower, about 0.10 mag airmass ${ }^{-1}$. Let $\Delta$ be the difference of the instrumental magnitudes and the catalog magnitudes of stars of known brightness. If the extinction at Dome $\mathrm{A}$ is comparable to that at Maunakea, over the GASC FOV we would expect $\Delta$ to exhibit a range versus air mass of roughly $0.06 \mathrm{mag}$ in the $B$-band, $0.04 \mathrm{mag}$ in the $V$-band, and $0.03 \mathrm{mag}$ in the $R$-band. No effect caused by the range of airmass has been detected with GASC data given its photometric accuracy, indicating a smaller atmospheric extinction coefficient at Dome A Antarctica compared to Maunakea.

We used the "pseudo-star" described in Section 4.4.2 as an indicator of the relative transparency variations to derive the likelihood of cloud cover at Dome A during the 2009 winter season. The reduction in transparency could be due to clouds, seasonal atmospheric variations, or even ice formed on the entrance transmission window. Some of those pairs of effects can hardly be separated, as they produce the same effect in the change of the transparency. Therefore, our results represent the upper limits to the cloud cover. Figure 15 shows the transparency and the estimated cloud cover during the 2009
Table 5

Sun Elevation Angles Corresponding to Increased Sky Brightness

\begin{tabular}{lccc}
\hline \hline Flux Increase & $B$ & $V$ & $R$ \\
\hline $20 \%$ & $-17^{\circ} .2$ & $-15^{\circ} .0$ & -14.7 \\
$50 \%$ & -16.0 & -13.9 & -13.6 \\
$100 \%$ & $-15^{\circ} .1$ & -13.1 & -12.7 \\
$200 \%$ & -14.2 & -12.2 & -11.9 \\
\hline
\end{tabular}

winter season. A long-term variation in transparency inferred from the brightness of the "pseudo-star" is unlikely due to cloud coverage, but is more likely attributable to a seasonal variation of the atmosphere above Dome A. A fifth-order polynomial has been used to fit this long-term trend, and the residuals were used to calculate the upper limit of the cloud coverage. The estimation of the cloud coverage is also based on the "pseudo-star" after applying a correction to this long-term variation. The brightest values of the "pseudo-star" indicate very clear sky with cloud coverage estimated to be 0 , and the reduction of the "pseudo-star" magnitude, defined as $\Delta m$, was correlated with the cloud coverage as follows:

$$
\Delta m=-2.5 \log \frac{\text { flux }_{1}}{\text { flux }_{2}}=-2.5 \log (1-\text { cloud cover }) .
$$

We find that the seasonal transparency degraded after 2009 June, during which the Sun was farthest below the horizon for the year. This agrees with Zou et al. (2010) to some extent. However, the possibility that such a long-term transparency variation is due to a change in the condition of the instrument cannot be ruled out. Table 6 gives the cloud coverage percentages at Dome A from 2009 May 19 to September 18. A rough comparison with the cloud coverage at Maunakea is given in Table 7 . This includes the cloud cover measured at the Gemini north Telescope and measurements with CSTAR in the I-band at Dome A during the 2008 winter season (Zou et al. 2010). CSTAR pointed at the SCP with an FOV of diameter 4.5 while the GASC FOV was $85^{\circ}$. The results from 2008 and 2009 are comparable. At Dome A it is "cloudy" or worse $2 \%-3.5 \%$ of the time, while at Maunakea, this number is much higher, $30 \%$. At Dome A there is less than 0.3 mag of extinction $62 \%-67 \%$ of the time, while at Maunakea the sky is photometric only $50 \%$ of the time.

A simple but effectively reliable way to check the cloud coverage estimated from the "pseudo-star" is to look at the original frames for certain fractions of cloud cover. Figure 16 presents four sample images of cloud coverage of $0 \%, 20 \%$, $70 \%$, and $95 \%$ obtained on 2009 June 26 at 01:16:22, 04:10:56, 18:23:18, and 20:54:41 UT. Many images estimated to have high cloud cover in GASC data did not show obvious cloudy patches. Instead, they showed a reduction in transparency over the entire FOV. It is hard to determine whether those extremely low transparency events were due to the sky or ice formation 


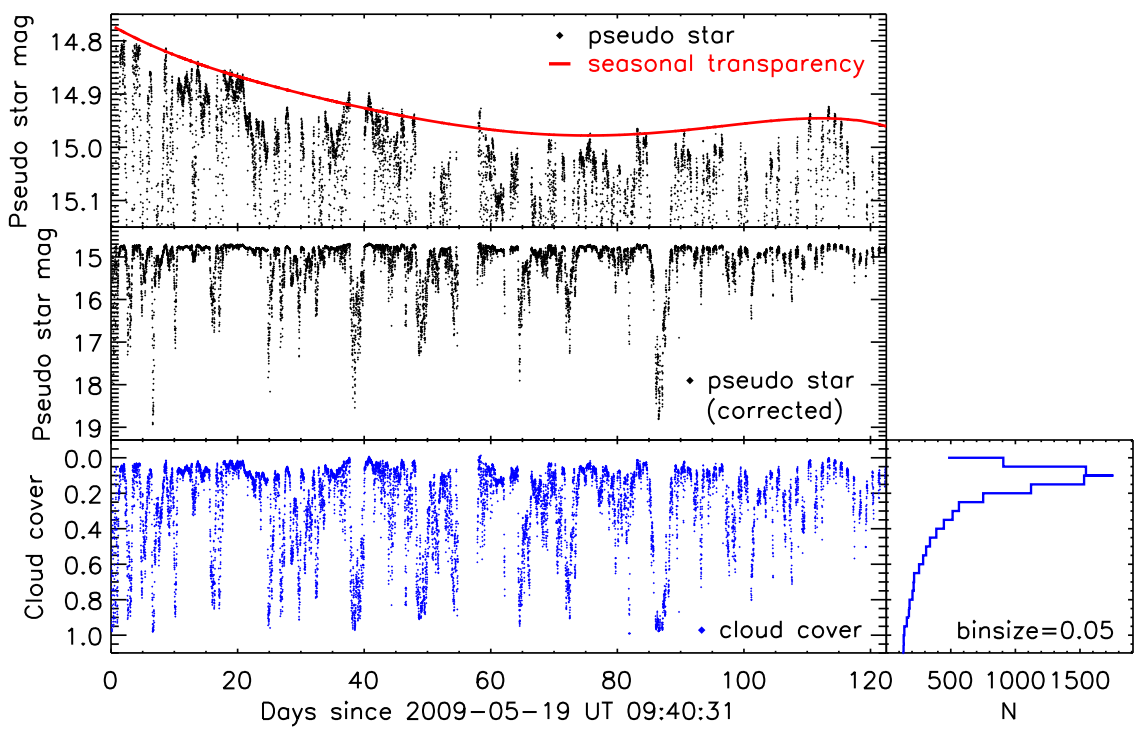

Figure 15. Atmospheric transparency estimated from the "pseudo-star" after correction for the long-term transparency variations. The black dots in the top panel are intentionally plotted with a small range of brightness of the pseudo-star. The red curve is a polynomial fit to the upper envelope and shows a long-term trend in the atmospheric transparency. The middle panel shows the variation of the "pseudo-star" after removing the seasonal transparency variation. The lower panel shows the time-series diagram of the implied cloud cover, with a histogram of the cloud cover data on the right. All magnitudes are uncalibrated instrumental magnitudes.
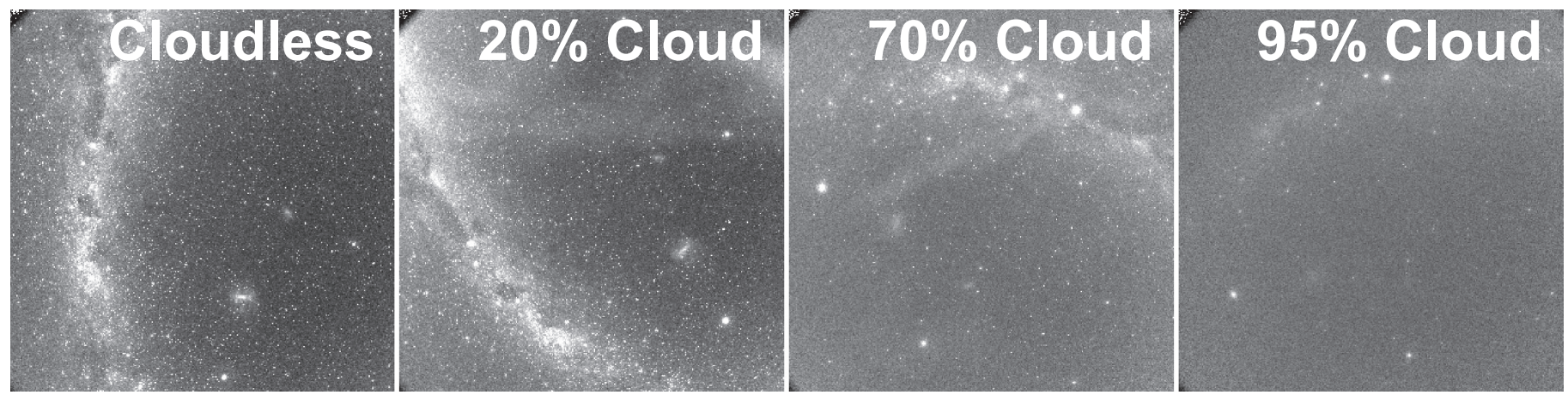

Figure 16. Four sample images showing cloudless sky, $20 \%$ cloud cover, $70 \%$ cloud cover, and $95 \%$ cloud cover, from left to right, respectively.

Table 6

Cloud Cover at Dome A

\begin{tabular}{|c|c|c|c|c|c|}
\hline Flux & Extinction (mag) & GASC2009 & GASC2009 ${ }^{\mathrm{a}}$ & Cstar2008 & Description \\
\hline$<50 \%$ & $>0.75$ & $17.2 \%$ & $19.9 \%$ & $9 \%$ & Thick \\
\hline $50 \%-75 \%$ & $0.31-0.75$ & $19.4 \%$ & $27.2 \%$ & $17 \%$ & Intermediate \\
\hline $75 \%-90 \%$ & $0.11-0.31$ & $29.1 \%$ & $42.1 \%$ & $23 \%$ & Thin \\
\hline$>90 \%$ & $<0.11$ & $34.3 \%$ & $10.8 \%$ & $51 \%$ & Little or none \\
\hline
\end{tabular}

Note.

${ }^{\mathrm{a}}$ Values obtained without correcting for the long-term transparency variation.

Table 7

Cloud Cover Compared to Maunakea

\begin{tabular}{|c|c|c|c|c|c|}
\hline Description & Extinction (mag) & Maunakea & Dome A (GASC2009) & Dome A (GASC2009) ${ }^{\mathrm{a}}$ & Dome A (Cstar2008) \\
\hline Any other usable & $>3$ & $10 \%$ & $1.0 \%$ & $1.1 \%$ & $0 \%$ \\
\hline Cloudy & $2-3$ & $20 \%$ & $2.5 \%$ & $2.8 \%$ & $2 \%$ \\
\hline Patchy cloud & $0.3-2$ & $20 \%$ & $34.2 \%$ & $45.1 \%$ & $31 \%$ \\
\hline Photometric & $<0.3$ & $50 \%$ & $62.4 \%$ & $51.0 \%$ & $67 \%$ \\
\hline
\end{tabular}

Note.

${ }^{\text {a }}$ Values obtained without correcting for the long-term transparency variation. 


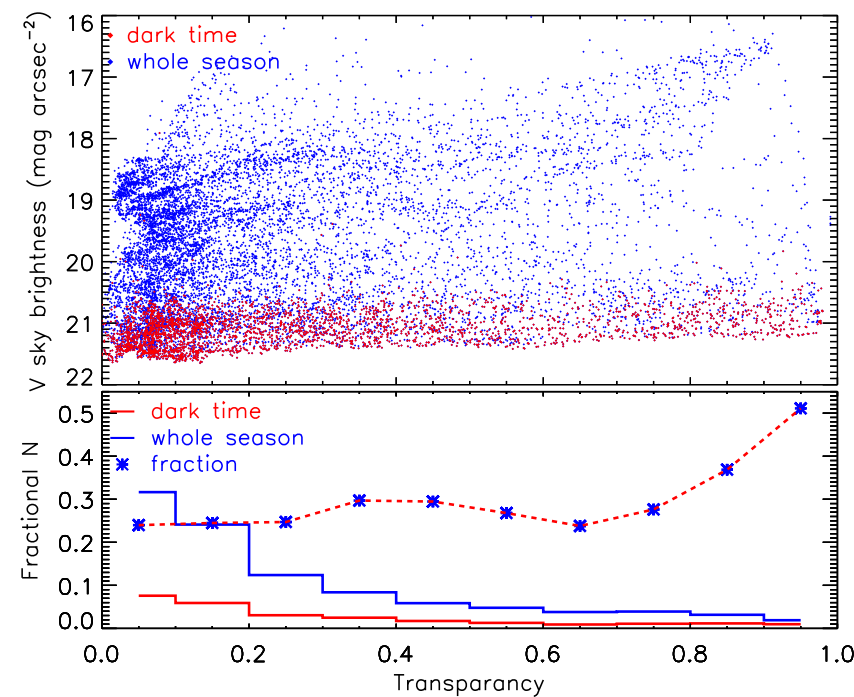

Figure 17. $V$-band sky brightness derived from the median ADUs within a $20^{\circ}$ circle centered at the SCP vs. the transparency (upper panel). The blue and red dots represent the sky brightness for the entire season and during the dark time, respectively. The lower panel shows the normalized histograms for the $V$-band sky brightness. The blue asterisks with red dashed lines show the ratio of the bin counts of the two histograms. The bottom panel shows that the transparency is independent of the sky brightness in seasonal statistics

on the entrance window. However, we can look at the sky brightness and the transparency estimated by the pseudo-star to see whether the estimation of transparency has biased the sky background. Figure 17 shows the transparency-sky brightness diagram. The lower panel shows that the transparency is independent of the sky brightness in seasonal statistics, indicating that our estimation of the cloud coverage based on the pseudo-star is not biased by the different sky backgrounds.

\subsection{Example Light Curves for Bright Stars}

High-precision, high-cadence time-series photometry serves as one of the major technical requirements for conducting asteroseismology. The search for exoplanets also benefits from high-quality photometric monitoring of stars. Stars within a magnitude range of $\sim 8$ to $\sim 15$ can be measured with $\sim 10 \mathrm{~cm}$ class and larger telescopes. However, uninterrupted monitoring of stars that are even brighter, i.e., magnitude 3-7, has not been feasible for previous Antarctic observations due to the very short time to reach the saturation level of a detector.

Our "ring correction" technique allows us to obtain a dispersion level of $\sim 0.03 \mathrm{mag}$ for stars around $5.5 \mathrm{mag}$ in four consecutive days. This valuable long-term, multicolor, consecutive photometric data set allows the study of eclipsing binaries, Cepheids, and other stellar variables. In Figure 18, we briefly present example light curves for a bright eclipsing binary $\zeta$ Phoenicis and a W Vir-type Cepheid variable $\kappa$ Pavonis with a short (4 day) and a long (120 day) period, respectively. More than 60 variables have been monitored by the GASC in the $B-, V$-, and $R$-bands. The multiband photometric studies of these bright variables will be presented in another paper.

\section{Conclusions}

In 2009, the GASC was deployed at Dome A in Antarctica to monitor the sky background and the variation of atmospheric transparency, and to perform photometry of bright targets in the field with an unprecedented window function. About 36,000 scientific images with $100 \mathrm{~s}$ exposure time, covering the Bessell $B, V$, and $R$ photometric bands have been used to quantify the $B-, V$-, and $R$-band sky brightness, and to estimate the upper limit of cloud coverage. In a subsequent paper, we shall present photometry of more than 60 bright stars in our FOV that shows significant variability based on GASC data after applying the method we developed to correct for the systematic error.

The median value of the sky brightness when the Sun elevation is less than $-18^{\circ}$ and the Moon is below the horizon is $22.45 \mathrm{mag} \operatorname{arcsec}^{-2}$ for the $B$-band, $21.40 \mathrm{mag} \mathrm{arcsec}^{-2}$ for the $V$-band, and $20.56 \mathrm{mag} \operatorname{arcsec}^{-2}$ for the $R$-band. If we consider a cumulative probability distribution, for the darkest $10 \%$ of the time, the $B-, V$-, and $R$-band sky brightness is 22.98 , 21.86 , and $21.68 \mathrm{mag} \operatorname{arcsec}^{-2}$, respectively. These are comparable to the values obtained at solar minimum at other best astronomical sites such as Maunakea and the observatories in northern Chile. For future instruments that will be operating at Dome A, customized filters or high spectral resolution designs could easily obtain better values on a more routine basis. A test carried out with GASC at Palomar Observatory indicated that the GASC "ring correction" method agrees with the Palomar NSBM within $0.12 \mathrm{mag} \operatorname{arcsec}^{-2}$. At Dome A, the sky brightness is quite constant within $30^{\circ}$ of the SCP.

A "pseudo-star" was constructed based on all the stars over the FOV as an indicator of transparency variations. The cloud coverage during the 2009 winter season has been estimated. We found that the seasonal transparency worsened in June. The transparency changed considerably in June and July when the Sun was at its lowest below the horizon for the year. About $63 \%$ of the time there was little or thin cloud coverage, using the same criteria for the cloud coverage adopted at the Gemini north Observatory at Maunakea, and also the cloud coverage estimation from CSTAR (Zou et al. 2010).

Solar and lunar models for the flux contributions to the sky background have been fitted, and the different flux enhancements in the sky background for different bandpasses have been obtained. Aurorae and airglow are hard to quantify with GASC observations due to limited photometric accuracy and unexpected instrumental effects. A visual inspection of the sky background after removing the solar and lunar contributions indicates a very limited effect of auroral events during the recent solar minimum.

We thank Shri Kulkarni and Caltech Optical Observatories, Gerard Van Belle, and Chas Beichman for their financial contributions to this project. We are grateful to Xiaofeng Wang, Chao Wu, Ming Yang, Tianmeng Zhang, Yanping Zhang, and Jilin Zhou for helpful discussions. This research is supported by the Chinese PANDA International Polar Year project and the Polar Research Institute of China. The project was funded by the following awards from the National Science Foundation Office of Polar Programs: ANT 0836571, ANT 0909664, and ANT 1043282. The project was also supported by the Strategic Priority Research Program "The Emergence of Cosmological Structures" of the Chinese Academy of Sciences, Grant No. XDB09000000. J.N.F. acknowledges the support from the Joint Fund of Astronomy of the National Natural Science Foundation of China (NSFC) and Chinese Academy of Sciences through the grant U1231202, the NSFC grant 11673003, the National Basic Research Program of China (973 Program 2014CB845700 and 2013CB834900), and the 

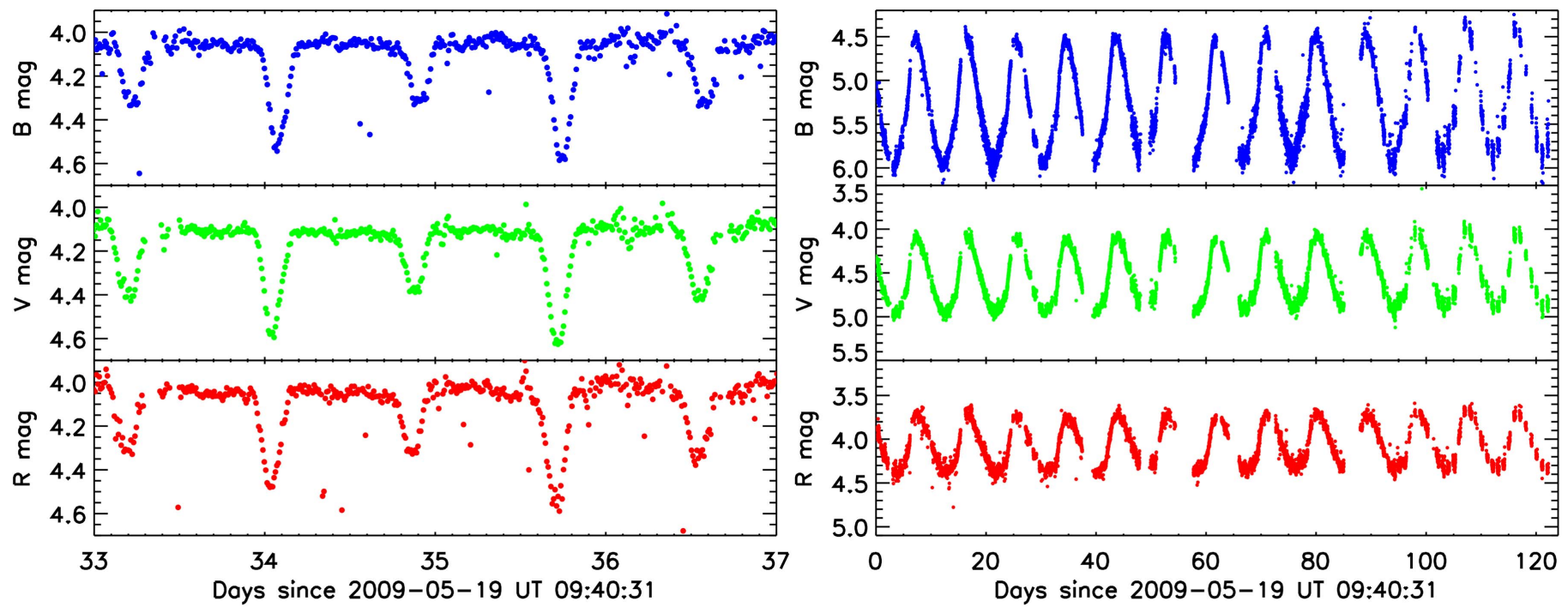

Figure 18. $B$-, $V$-, and $R$-band light curves for an eclipsing binary $\zeta$ Phoenicis (left panel) and a W Vir-type Cepheid variable $\kappa$ Pavonis (right panel).

LAMOST Fellowship supported by the Special Funding for Advanced Users, budgeted and administrated by the Center for Astronomical Mega-Science, Chinese Academy of Sciences (CAMS). The operation of PLATO at Dome A is supported by the Australian Research Council, the Australian Antarctic Division, and the University of New South Wales. The authors wish to thank all the members of the 2008/2009/2010 PRIC Dome A heroic expeditions.

\section{References}

Agabi, A., Aristidi, E., Azouit, M., et al. 2006, PASP, 118, 344 Aristidi, E., Fossat, E., Agabi, A., et al. 2009, A\&A, 499, 955 Aristidi, E., Vernin, J., Fossat, E., et al. 2015, MNRAS, 454, 4304 Ashley, M. C. B., Burton, M. G., Storey, J. W. V., et al. 1996, PASP, 108, 721 Baglin, A., Auvergne, M., Boisnard, L., et al. 2006, in COSPAR Meeting 36 , 36th COSPAR Scientific Assembly, 3749

Benn, C. R., \& Ellison, S. L. 1998, NewAR, 42, 503

Bessell, M. S. 1990, PASP, 102, 1181

Bonner, C. S., Ashley, M. C. B., Cui, X., et al. 2010, PASP, 122, 1122

Borucki, W. J., Koch, D., Basri, G., et al. 2010, Sci, 327, 977

Chadid, M., Vernin, J., Abe, L., et al. 2016, Proc. SPIE, 9908, 99080T

Chadid, M., Vernin, J., Mekarnia, D., et al. 2010, A\&A, 516, L15

Chadid, M., Vernin, J., Preston, G., et al. 2014, AJ, 148, 88

Garstang, R. H. 1991, PASP, 103, 1109

Gattinger, R. L., \& Jones, A. V. 1974, CaJPh, 52, 2343

Gehrels, N., Chincarini, G., Giommi, P., et al. 2004, ApJ, 611, 1005

Giordano, C., Vernin, J., Chadid, M., et al. 2012, PASP, 124, 494

Huang, Z., Fu, J., Zong, W., et al. 2015, AJ, 149, 25

Jones, A. V., \& Gattinger, R. L. 1975, CaJPh, 53, 1806

Jones, D. O., Rodney, S. A., Riess, A. G., et al. 2013, ApJ, 768, 166

Kenyon, S. L., \& Storey, J. W. V. 2006, PASP, 118, 489

Krisciunas, K. 1997, PASP, 109, 1181

Krisciunas, K., Bogglio, H., Sanhueza, P., \& Smith, M. G. 2010, PASP, 122, 373

Krisciunas, K., \& Schaefer, B. E. 1991, PASP, 103, 1033

Krisciunas, K., Semler, D. R., Richards, J., et al. 2007, PASP, 119, 687

Krisciunas, K., Sinton, W., Tholen, K., et al. 1987, PASP, 99, 887

Landolt, A. U. 1992, AJ, 104, 340
Lawrence, J. S. 2004, PASP, 116, 482

Lawrence, J. S., Ashley, M. C. B., Tokovinin, A., \& Travouillon, T. 2004, Natur, 431, 278

Li, G., Fu, J., \& Liu, X. 2015, arXiv:1510.06134

Liang, E.-S., Wang, S., Zhou, J.-L., et al. 2016, arXiv:1608.07904

Liu, Y., Zhou, X., Sun, W.-H., et al. 2003, PASP, 115, 495

Marks, R. D. 2002, A\&A, 385, 328

Marks, R. D., Vernin, J., Azouit, M., et al. 1996, A\&AS, 118, 385

McKenna, D. 2008, http://www.sao.arizona.edu/FLWO/SBM/SBMreport_ McKenna_Apr08.pdf

Meng, Z., Zhou, X., Zhang, H., et al. 2013, PASP, 125, 1015

Moore, A., Allen, G., Aristidi, E., et al. 2008, Proc. SPIE, 7012, 701226

Nguyen, H. T., Rauscher, B. J., Severson, S. A., et al. 1996, PASP, 108, 718

Oelkers, R. J., Macri, L. M., Wang, L., et al. 2015, AJ, 149, 50

Patat, F. 2003, A\&A, 400, 1183

Rau, A., Kulkarni, S. R., Law, N. M., et al. 2009, PASP, 121, 1334

Rayleigh, L. 1928, RSPSA, 119, 11

Rayleigh, L., \& Jones, H. S. 1935, RSPSA, 151, 22

Roach, F. E., \& Gordon, J. L. 1973, The Light of the Night Sky (Dordrecht: Reidel)

Roming, P. W. A., Kennedy, T. E., Mason, K. O., et al. 2005, SSRv, 120, 95 Saunders, W., Lawrence, J. S., Storey, J. W. V., et al. 2009, PASP, 121, 976 Sims, G., Ashley, M. C. B., Cui, X., et al. 2010, Proc. SPIE, 7733, 77334M

Sims, G., Ashley, M. C. B., Cui, X., et al. 2012a, PASP, 124, 637

Sims, G., Ashley, M. C. B., Cui, X., et al. 2012b, PASP, 124, 74

Vernin, J., Chadid, M., Aristidi, E., et al. 2009, A\&A, 500, 1271

Walker, M. F. 1988, PASP, 100, 496

Wang, L., Macri, L. M., Krisciunas, K., et al. 2011, AJ, 142, 155

Wang, L., Macri, L. M., Wang, L., et al. 2013, AJ, 146, 139

Wang, S., Zhang, H., Zhou, J.-L., et al. 2014a, ApJS, 211, 26

Wang, S., Zhang, H., Zhou, X., et al. 2015, ApJS, 218, 20

Wang, S., Zhou, X., Zhang, H., et al. 2012, PASP, 124, 1167

Wang, S.-H., Zhou, X., Zhang, H., et al. 2014b, RAA, 14, 345

Yang, H., Allen, G., Ashley, M. C. B., et al. 2009, PASP, 121, 174

Yang, H., Kulesa, C. A., Walker, C. K., et al. 2010, PASP, 122, 490

Yang, M., Zhang, H., Wang, S., et al. 2015, ApJS, 217, 28

Yuan, X., Cui, X., Gu, B., et al. 2014, Proc. SPIE, 9145, 91450F

Yuan, X., Cui, X., Liu, G., et al. 2008, Proc. SPIE, 7012, 70124G

Zong, W., Fu, J.-N., Niu, J.-S., et al. 2015, AJ, 149, 84

Zou, H., Zhou, X., Jiang, Z., et al. 2010, AJ, 140, 602 